\title{
THE SYMPLECTIC GEOMETRY OF POLYGONS IN EUCLIDEAN SPACE
}

\author{
MICHAEL KAPOVICH \& JOHN J. MILLSON
}

\begin{abstract}
We study the symplectic geometry of moduli spaces $M_{r}$ of polygons with fixed side lengths in Euclidean space. We show that $M_{r}$ has a natural structure of a complex analytic space and is complex-analytically isomorphic to the weighted quotient of $\left(S^{2}\right)^{n}$ by $P L S(2, \mathbb{C})$ constructed by Deligne and Mostow. We study the Hamiltonian flows on $M_{r}$ obtained by bending the polygon along diagonals and show the group generated by such flows acts transitively on $M_{r}$. We also relate these flows to the twist flows of Goldman and Jeffrey-Weitsman.
\end{abstract}

\section{Introduction}

Let $\mathcal{P}_{n}$ be the space of all $n$-gons with distinguished vertices in Euclidean space $\mathbb{E}^{3}$. An $n$-gon $P$ is determined by its vertices $v_{1}, \ldots, v_{n}$. These vertices are joined in cyclic order by edges $e_{1}, \ldots, e_{n}$ where $e_{i}$ is the oriented line segment from $v_{i}$ to $v_{i+1}$. Two polygons $P=\left(v_{1}, \ldots, v_{n}\right)$ and $Q=\left(w_{1}, \ldots, w_{n}\right)$ are identified if and only if there exists an orientation preserving isometry $g$ of $\mathbb{E}^{3}$ which sends the vertices of $P$ to the vertices of $Q$, that is

$$
g v_{i}=w_{i}, 1 \leq i \leq n
$$

Let $r=\left(r_{1}, \ldots, r_{n}\right)$ be an $n$-tuple of positive real numbers. Then $M_{r}$ is defined to be the space of $n$-gons with side lengths $r_{1}, \ldots, r_{n}$ modulo isometries as above. The group $\mathbb{R}_{+}$acts on $\mathcal{P}_{n}$ by scaling and we obtain an induced isomorphism $M_{r} \cong M_{\lambda r}$ for $\lambda \in \mathbb{R}_{+}$. Thus we lose nothing by assuming

$$
\sum_{i=1}^{n} r_{i}=2
$$

We make this normalization to agree with [4], §2.

Received October 17, 1994, and, in revise form, October 28, 1995. The first author was partially supported by NSF grant DMS-9306140 at University of Utah and the second author by NSF grant DMS-9205154, the University of Maryland. 
We observe that the map

$$
\pi: \mathcal{P}_{n} \rightarrow \mathbb{R}_{+}^{n}
$$

which assigns the vector $r$ of side lengths to an $n$-gon $P$ has image the polyhedron $\mathcal{D}_{n}$ of [10], $\S 2$. The moduli spaces $M_{r}$ then appear as the fibers of $\pi$ and their topology may be obtained by the wall- crossing arguments of [10] and [21]. We recall that the moduli space $M_{r}$ is a smooth manifold iff $M_{r}$ does not contain a degenerate polygon. We denote by $\Sigma$ the collection of hyperplanes in $\mathcal{D}_{n}$ described in [10], then $M_{r}$ is singular iff $r \in \Sigma$.

This paper is concerned with the symplectic geometry of the space $M_{r}$. We prove two main results. The space $\left(S^{2}\right)^{n}$ is given the symplectic structure

$$
\sum_{i=1}^{n} r_{i} v o l
$$

where $v o l$ is the standard symplectic structure on $S^{2}$. Our first result gives a natural isomorphism from $M_{r}$ to the weighted quotient of $\left(S^{2}\right)^{n}$ by $P L S(2, \mathbb{C})$ for the weights $r_{1}, \ldots, r_{n}$ constructed by Deligne and Mostow in [4]. The construction goes as follows. The closing condition

$$
e_{1}+\ldots .+e_{n}=0
$$

for the edges of the polygon $P$ defines the zero level set for the momentum map for the diagonal action of $S O(3)$ on $\left(S^{2}\right)^{n}$. Thus $M_{r}$ is the weighted symplectic quotient of $\left(S^{2}\right)^{n}$ by $S O(3)$. We give $M_{r}$ the structure of a complex-analytic space with at worst quadratic singularities. This is immediate at smooth points of $M_{r}$ since $\left(S^{2}\right)^{n}$ is Kähler. However it requires more effort at the singular points.

We then extend the Kirwan-Kempf-Ness theorem [12], [13], [19] to show that the complex- analytic quotient constructed by Deligne and Mostow is complex analytically isomorphic to the symplectic quotient. We observe that the cusp points $Q_{\text {cusp }}$ of [4] correspond to the singular points of $M_{r}$ which in turn correspond to degenerate polygons, i.e., polygons that lie in a line.

Our second main result is the construction of "bending flows". Suppose $P$ has edges $e_{1}, \ldots, e_{n}$. We let

$$
\mu_{k}=e_{1}+\ldots .+e_{k+1}, 1 \leq k \leq n-3
$$

be the $k$-th diagonal of $P$ and define functions

$$
f_{1}, \ldots, f_{n-3}
$$


on $M_{r}$ by

$$
f_{k}(P)=\frac{1}{2}\left\|\mu_{k}\right\|^{2}
$$

We check that the functions $f_{k}$ Poisson commute and that the corresponding Hamiltonian flows have periodic orbits. Since $\operatorname{dim} M_{r}=2 n-6$ we see that $M_{r}$ is completely integrable and is almost a toric varietyunfortunately we cannot normalize the flows to have constant periods if the functions $f_{k}$ have zero's on $M_{r}$. The Hamiltonian flow for $f_{k}$ has the following geometric description. Construct a polyhedral surface $S$ bounded by $P$ by filling in the triangles

$$
\Delta_{1}, \Delta_{2}, \ldots, \Delta_{n-2}
$$

where $\Delta_{k}$ has edges $\mu_{k} \neq 0, e_{k+1}, \mu_{k+1}, 1 \leq k \leq n-1$ (we have $\mu_{n-1}=$ $\left.e_{n}\right)$. Some of these triangles may be degenerate. The diagonal $\mu_{k}$ divides $S$ into two pieces. Keep the second piece fixed and rotate the first piece around the diagonal with angular velocity equal to $f_{k}(P)$. Thus the surface $S$ is "bent" along the diagonal and we call our flows bending flows. If $\mu_{k}=0$ then $P$ is fixed by the flow. However the bending flow does not preserve the complex structure of $M_{r}$.

Let $M_{r}^{\prime}$ denote the dense open subset of $M_{r}$ consisting of those polygons $P$ such that none of the above diagonals have zero length. Thus $M_{r}^{\prime}$ contains all the embedded polygons. Then the functions $\ell_{i}=\sqrt{2 f_{i}}$, $1 \leq i \leq n-3$, are smooth on $M_{r}^{\prime}$. The resulting flows are similar to those above except they have constant periods. We obtain a Hamiltonian action of an $n-3$-torus $T$ on $M_{r}^{\prime}$ by bending as above. If we further restrict to the dense open subset $M_{r}^{0} \subset M_{r}^{\prime}$ consisting of those polygons so that $\mu_{i}$ and $e_{i+1}$ are not collinear, $1 \leq i \leq n$, then we can introduce "action-angle" coordinates on $M_{r}^{0}$. Note that under the above hypothesis none of the triangles $\Delta_{i}$ is degenerate. We let $\hat{\theta}_{i} \in \mathbb{R} / 2 \pi \mathbb{Z}$ be the oriented dihedral angle between $\Delta_{i}$ and $\Delta_{i+1}$. In $\S 4$ we prove that

$$
\theta_{1}=\pi-\hat{\theta}_{1}, \ldots, \theta_{n-3}=\pi-\hat{\theta}_{n-3}, \ell_{1}, \ldots, \ell_{n-3}
$$

are action-angle variables.

In Section 5 we relate our results on bending to the "twist" deformations of [7], [9] and [22]. Our main new contribution here is the discovery of an invariant, nondegenerate symmetric bilinear form on the Lie algebra of the group of Euclidean motions. Also in Proposition 5.8 we give a general formula for the symplectic structure on the space of relative deformations of a flat $G$-bundle over an $n$ times punctured sphere. Here we assume that the Lie algebra $\mathcal{G}$ of $G$ admits a non-degenerate, $G$-invariant symmetric bilinear form. 
In $\S 6$ we show that the subgroup of the symplectic diffeomorphisms of $M_{r}$ generated by bendings on the diagonals of $\mathrm{P}$ acts transitively on $M_{r}$. In Figure 1 we show how to bend a square into a parallelogram.

It is a remarkable fact that most of the results (and arguments) of this paper generalize to the space of smooth isometric maps from $S^{1}$ with a fixed Riemannian metric to $\mathbb{E}^{3}$ modulo proper Euclidean motions, i.e., to regular $\infty$-gons. These results will appear in [16] and [17].

After this paper was submitted for publication we received the paper [14] by A. Klyachko . Klyachko also discovered a Kähler structure on $M_{r}$ and that it is biholomorphically equivalent to the configuration space $\left(S^{2}\right)^{n} / P S L(2, \mathbb{C})$, however he did not use the conformal center of mass construction and did not give a proof of this equivalence. Otherwise, the main emphasis of [14] is on construction of a cell-decomposition of $M_{r}$ and calculation of (co)homological invariants of this space; [14] does not contain the geometric interpretation and transitivity of bending flows, action-angle coordinates and connections with gauge theory.

\section{Moduli of polygons and weighted quotients of configuration spaces of points on the sphere}

Our goal in this section is to give $M_{r}$ the structure of a complex analytic space and to construct a natural complex analytic equivalence from $M_{r}$ to $Q_{s s t}$, the weighted quotient of the configuration space of $n$ points on $S^{2}$ by $P S L(2, \mathbb{C})$ constructed in [4], $\S 4$. We define a subspace $\tilde{\mathcal{M}}_{r} \subset\left(S^{2}\right)^{n}$ by

$$
\tilde{\mathcal{M}}_{r}=\left\{u \in\left(S^{2}\right)^{n}: \sum_{j=1}^{n} r_{j} u_{j}=0\right\}
$$

Each polygon $P$ in the moduli space $M_{r}$ corresponds (up to translation) to the collection of vectors

$$
\left(e_{1}, \ldots, e_{n}\right) \in\left(\mathbb{R}^{3}-\{0\}\right)^{n}
$$

The normalized vectors $u_{j}=e_{j} / r_{j}$ belong to the sphere $S^{2}$. The polygon $P$ is defined up to a Euclidean isometry, therefore the vector

$$
\vec{u}=\left(u_{1}, \ldots, u_{n}\right)
$$

is defined up to rotation around zero. Since the polygon $P$ is closed we conclude that

$$
\sum_{j=1}^{n} r_{j} u_{j}=0
$$


Thus there is a natural homeomorphism

$$
\epsilon: M_{r} \rightarrow \mathcal{M}_{r}=\tilde{\mathcal{M}}_{r} / S O(3)
$$

We will call $\epsilon$ the Gauss map.

We now review the definition of the weighted quotient $Q_{s s t}$ of the configuration space of $n$ points on $S^{2}$ following [4], §4. Let $M \subset\left(S^{2}\right)^{n}$ be the set of $n$-tuples of distinct points. Then $Q=M / P S L(2, \mathbb{C})$ is a Hausdorff complex manifold.

Definition 2.1. A point $\vec{u} \in\left(S^{2}\right)^{n}$ is called $r$-stable (resp. semistable) if

$$
\sum_{u_{j}=v} r_{j}<1(\text { resp. } \leq 1)
$$

for all $v \in S^{2}$. The sets of stable and semi-stable points will be denoted by $M_{s t}$ and $M_{s s t}$ respectively. A semi-stable point $\vec{u} \in\left(S^{2}\right)^{n}$ is said to be a nice semi-stable point if it is either stable or the orbit $P S L(2, \mathbb{C}) \vec{u}$ is closed in $M_{s s t}$.

We denote the space of nice semi-stable points by $M_{n s s t}$. We have the inclusions

$$
M_{s t} \subset M_{n s t} \subset M_{s s t}
$$

Let $M_{\text {cusp }}=M_{s s t}-M_{s t}$. We obtain the points in $M_{\text {cusp }}$ in the following way. Partition $S=\{1, \ldots, n\}$ into two disjoint sets $S=S_{1} \cup S_{2}$ with $S_{1}=\left\{i_{1}, \ldots, i_{k}\right\}, S_{2}=\left\{j_{1}, . ., j_{n-k}\right\}$ in such a way that $r_{i_{1}}+\ldots+r_{i_{k}}=1$ (whence $r_{j_{1}}+\ldots+r_{j_{n-k}}=1$ ). Then $\vec{u}$ is in $M_{\text {cusp }}$ if either $u_{i_{1}}=\ldots=u_{i_{k}}$ or $u_{j_{1}}=\ldots=u_{j_{n-k}}$. The reader will verify that $\vec{u} \in M_{\text {cusp }}$ is a nice semi-stable point if and only if both sets of the equations above hold. All points in $M_{\text {cusp }}$ are obtained in this way. Clearly the nice semistable points correspond under $\epsilon^{-1}$ to the degenerate polygons with $S_{1}$ determined by the forward-tracks and $S_{2}$ by the back-tracks. On $M_{s s t}$ we define a relation $\mathcal{R}$ via:

$\vec{u} \equiv \vec{w}(\bmod \mathcal{R})$ if either

(a) $\vec{u}, \vec{w} \in M_{s t}$ and $\vec{w} \in P S L(2, \mathbb{C}) \vec{u}$, or

(b) $\vec{u}, \vec{w} \in M_{\text {cusp }}$ and the partitions of $S$ corresponding to $\vec{u}, \vec{w}$ coincide.

The reader will verify that if $\vec{u}, \vec{w} \in M_{n s s t}$ then $\vec{u} \equiv \vec{w}(\bmod \mathcal{R})$ if and only if $\vec{w} \in P S L(2, \mathbb{C}) \vec{u}$.

It is clear that $\mathcal{R}$ is an equivalence relation. Set

$$
Q_{s s t}=M_{s s t} / \mathcal{R}, Q_{n s s t}=M_{n s s t} / \mathcal{R}, Q_{s t}=M_{s t} / \mathcal{R}, Q_{\text {cusp }}=M_{\text {cusp }} / \mathcal{R}
$$

each with the quotient topology. The elements of $Q_{c u s p}$ are uniquely determined by their partitions. Thus $Q_{\text {cusp }}$ is a finite set. It is clear 
that each equivalence class in $Q_{\text {cusp }}$ contains a unique $P S L(2, \mathbb{C})$-orbit of nice semi-stable points whence the inclusion

$$
M_{n s s t} \subset M_{s s t}
$$

induces an isomorphism

$$
Q_{n s s t}=M_{n s s t} / P S L(2, \mathbb{C}) \rightarrow Q_{s s t}
$$

In case $r_{1}, \ldots, r_{n}$ are rational then the quotient space $Q_{s s t}$ can be given a structure of an algebraic variety by the techniques of geometric invariant theory applied to certain equivariant projective embedding of $\left(S^{2}\right)^{n}$, see [4], §4.6. This concludes our review of [4], §4. We now establish the connection with the moduli space $M_{r}$.

We recall several basic definitions from symplectic geometry. Suppose that $N$ is a simply-connected Kähler manifold with symplectic form $\omega$ and $G^{c}$ is a complex reductive Lie group acting holomorphically on $N$. Let $G$ be a maximal compact subgroup in $G^{c}$. We may assume that $G$ acts symplectically. Then the Lie algebra $\mathcal{G}$ of $G$ maps naturally into the space of vector-fields on $N$. Each element $X \in \mathcal{G}$ defines a Hamiltonian $f_{X}: N \rightarrow \mathbb{R}$ so that $d f_{X}(Y)=\omega(X, Y)$ for every $Y \in T(N)$. There exists a map $\mu: N \rightarrow \mathcal{G}^{*}$ such that $\langle\mu(z), X\rangle=f_{X}(z)$. The map $\mu$ is called the momentum map for the action of $G$. The space $\mu^{-1}(0) / G$ is called the symplectic quotient of $N$ by $G$ to be denoted by $N / / G$.

Let $v o l$ be the $S O(3)$-invariant volume form on $S^{2}$ normalized by

$$
\int_{S^{2}} v o l=4 \pi
$$

Fix a vector $r=\left(r_{1}, \ldots, r_{n}\right)$ with positive entries. We give $\left(S^{2}\right)^{n}$ the symplectic form

$$
\omega=\sum_{j=1}^{n} r_{j} p_{j}^{*}(v o l)
$$

where $p_{j}^{*}:\left(S^{2}\right)^{n} \rightarrow S^{2}$ is the projection on the $j$-th factor. The maximal compact subgroup $S O(3) \subset P S L(2, \mathbb{C})$ acts symplectically on $\left(\left(S^{2}\right)^{n}, \omega\right)$. We let

$$
\mu:\left(S^{2}\right)^{n} \rightarrow \mathbb{R}^{3}
$$

be the associated momentum map.

Here we have identified the Lie algebra $s o(3)$ of $S O(3)$ with the space $\left(\mathbb{R}^{3}, \times\right)$ where $\times$ is the usual cross-product and $\mathbb{R}^{3} \cong\left(\mathbb{R}^{3}\right)^{*}$ via the Euclidean structure on $\mathbb{R}^{3}$. The identification $\left(\mathbb{R}^{3}, \times\right) \rightarrow s o(3)$ is given by $u \mapsto \operatorname{ad}_{u}$ where $\operatorname{ad}_{u}(v)=u \times v, u, v \in \mathbb{R}^{3}$.

Lemma 2.2 .

$$
\mu(\vec{u})=r_{1} u_{1}+\ldots+r_{n} u_{n}
$$


(compare Lemma 3.1)

Proof. First note that in case $n=1$ the momentum map $\mu: S^{2} \rightarrow \mathbb{R}^{3}$ for the symplectic structure $\rho \cdot v o l$ is given by

$$
\mu(u)=\rho \cdot u
$$

But the momentum map of a diagonal action on a product is the sum of the individual momentum maps.

Thus the space $\mathcal{M}_{r}$ is the symplectic quotient of $\left(S^{2}\right)^{n}$ (equipped with the symplectic structure $\omega$ defined above) by $S O(3)$, which is the subquotient $\mu^{-1}(0) / S O(3)$.

We obtain the following

Theorem 2.3. The Gauss map $\epsilon$ is a homeomorphism from the moduli space $M_{r}$ of $n$-gons in $\mathbb{R}^{3}$ with fixed side lengths to the weighted symplectic quotient of $\left(S^{2}\right)^{n}$ by $S O(3)$ acting diagonally.

We now prove that $M_{r}$ is a complex analytic space. Let $\Sigma \subset M_{r}$ be the subset of degenerate polygons ( $\Sigma$ is a finite collection of points). Then $M_{r}-\Sigma$ is the symplectic quotient of a Kähler manifold and is consequently a Kähler manifold [18], Ch. $8, \S 3$. It remains to give $M_{r}$ a complex structure in the neighborhood of a degenerate polygon.

To this end let $P$ be a degenerate $n$-gon which has $p+1$ "forwardtracks" and $q+1$ "back-tracks". The following lemma is a special case of [1], Corollary 4.2, except for the connection with the number of backtracks and forward-tracks. To establish this connection and for the sake of clarity we prove the following

Lemma 2.4. There is a neighborhood of $P$ in $M_{r}$ homeomorphic to the symplectic quotient $U / / S O(2)$ where $U$ is a neighborhood of 0 in $\mathbb{C}^{n-2}, S O(2)$ acts by symplectic isometries of the parallel symplectic form of $\mathbb{C}^{n-2}$ and is the Hamiltonian flow for the Hamiltonian $h$ : $\mathbb{C}^{n-2} \rightarrow \mathbb{R}$ given by the formula

$$
h\left(z_{1}, \ldots, z_{p}, w_{1}, \ldots, w_{q}\right)=\sum_{i=1}^{p}\left|z_{i}\right|^{2}-\sum_{i=1}^{q}\left|w_{i}\right|^{2}
$$

Proof. Let $\left\{\epsilon_{1}, \epsilon_{2}, \epsilon_{3}\right\}$ be the standard basis of $\mathbb{R}^{3}$. We may assume that $P$ is contained in the $x$-axis and that the last edge $e_{n}$ of $P$ is given by $e_{n}=r_{n} \epsilon_{1}$. We lift $\epsilon(P) \in \mathcal{M}_{r}$ to $\vec{u} \in\left(S^{2}\right)^{n}$ with

$$
\vec{u}=\left(\eta_{1} \epsilon_{1}, \eta_{2} \epsilon_{1}, \ldots, \eta_{n-1} \epsilon_{1}, \eta_{n} \epsilon_{1}\right)
$$

Here $\eta_{k} \in\{ \pm 1\}, \eta_{n}=1$, there are $p+1$ plus ones and $q+1$ minus ones and

$$
\sum_{k=1}^{n-1} \eta_{k} r_{k}=-r_{n}
$$


Our goal is to investigate the symplectic quotient of $\left(S^{2}\right)^{n}$ by $S O(3)$ near $\vec{u}$. We let $H \cong S O(2)$ be the subgroup of $S O(3)$ fixing $\epsilon_{1}$. Thus $H$ is the isotropy subgroup of $P$. We will often write $S O(2)$ instead $H$ in what follows.

We begin by constructing a slice $S$ through $\vec{u}$ for the action of $S O(3)$ on $\left(S^{2}\right)^{n}$. Define $S=\left\{\vec{s}=\left(s_{1}, \ldots, s_{n}\right) \in\left(S^{2}\right)^{n}: s_{n}=\epsilon_{1}\right\}$. Then $S$ is a smooth submanifold of $\left(S^{2}\right)^{n}$ of dimension $2 n-2$. It is immediate that $S$ satisfies the slice axioms:

- $h S \subset S, h \in H$;

- If $g S \cap S \neq \emptyset, g \in S O(3)$ then $g \in H$;

- The natural map $\alpha: S O(3) \times_{H} S \rightarrow\left(S^{2}\right)^{n}$ given by $\alpha([g, \vec{s}])=$ $g \vec{s}$ is a diffeomorphism.

We transfer the symplectic form $\omega$ from $\left(S^{2}\right)^{n}$ to $X=S O(3) \times_{H} S$. It is then immediate that the induced momentum map $\mu: X \rightarrow \mathbb{R}^{3}$ is given by

We define $S_{0} \subset S_{1} \subset S$ by

$$
\mu([g, \vec{s}])=g \sum_{i=1}^{n} r_{i} s_{i}
$$

$$
\begin{gathered}
S_{1}=\left\{\vec{s}=\left(s_{1}, \ldots, s_{n}\right) \in\left(S^{2}\right)^{n}: \sum_{i=1}^{n} r_{i} s_{i} \in \mathbb{R} \cdot \epsilon_{1}\right\} \\
S_{0}=\left\{\vec{s}=\left(s_{1}, \ldots, s_{n}\right) \in\left(S^{2}\right)^{n}: \sum_{i=1}^{n} r_{i} s_{i}=0\right\}
\end{gathered}
$$

We note that $\mu^{-1}(0)=S O(3) \times_{S O(2)} S_{0}$ and consequently the map $\alpha$ induces a homomorphism $\alpha: S_{0} / S O(2) \rightarrow\left(S^{2}\right)^{n} / / S O(3)$. We are done if we can prove that there is a neighborhood $V$ of $\vec{u}$ in $S_{0}$ such that $V / S O(2)$ is isomorphic to a neighborhood of 0 in the symplectic quotient $\mathbb{C}^{n-2} / / S O(2)$ described above.

We let $f: S \rightarrow \mathbb{R}^{3}$ be the map given by $f(\vec{s})=\sum_{i=1}^{n} r_{i} s_{i}$. We want to investigate $f^{-1}(0)$ near $\vec{u}$. Let $f_{1}, f_{2}, f_{3}$ be the components of $f$. Let $g=\left(f_{2}, f_{3}\right)$ whence $g: S \rightarrow \mathbb{R}^{2}$ and $g^{-1}(0)=S_{1}$. Since $d g_{u}: T_{u}\left(S^{2}\right) \rightarrow T_{0}\left(\mathbb{R}^{2}\right)$ is onto there is a neighborhood $V$ of $\vec{u}$ (which we may assume is $S O(2)$-invariant) such that $V \cap S_{1}$ is a smooth manifold of dimension $2 n-4$. Then

$$
V \cap S_{0}=\left\{\vec{s} \in V \cap S_{1}: f_{1}(\vec{s})=0\right\}
$$

We observe that $f_{1}=\mu \cdot \epsilon_{1}$ is the Hamiltonian function for the $S O(2)$ action on $S$. Clearly $S O(2)$ carries $S_{1}$ into itself.

Let $\rho$ denote the isotropy representation of $S O(2)$ on $T_{u}\left(V \cap S_{1}\right)$. We note that $\rho$ preserves the almost complex structure $J$ on $T_{u}\left(V \cap S_{1}\right)$ 
given by $J(\delta)=\vec{u} \times \vec{\delta}$ and $\rho$ preserves the parallel symplectic form $\omega_{\vec{u}}$ on $T_{\vec{u}}\left(S_{1}\right)$. After shrinking $V$ and applying Darboux's Theorem we may assume that the Riemannian exponential map $\exp _{\vec{u}}: T_{\vec{u}}\left(S_{1}\right) \rightarrow S_{1}$ induces a $S O(2)$-equivariant symplectic isomorphism from a neighborhood $U$ of 0 in $T_{\vec{u}}\left(S_{1}\right)$ to $V \cap S_{1}$. Thus $\exp _{\vec{u}}$ induces an isomorphism from $U / / S O(2)$ onto $V \cap S_{1} / / S O(2)$. We have accordingly reduced the problem to the linear case.

We have $S O(2)$-equivariant inclusions of symplectic vector spaces

$$
T_{\vec{u}}\left(S_{1}\right) \subset T_{\vec{u}}(S) \subset T_{\vec{u}}\left(\left(S^{2}\right)^{n}\right)
$$

where

$$
\begin{gathered}
T_{\vec{u}}(S)=\left\{\vec{\delta}: \delta_{i} \cdot \epsilon_{1}=0, \delta_{n}=0\right\} \\
T_{\vec{u}(}\left(S_{1}\right)=\left\{\vec{\delta}: \delta_{i} \cdot \epsilon_{1}=0, \delta_{n}=0, \sum_{i=1}^{n} r_{i} \delta_{i} \in \mathbb{R} \cdot \epsilon_{1}\right\}
\end{gathered}
$$

But since $\delta_{i}$ is orthogonal to the $x$-axis for all $i$, for $\vec{\delta} \in T_{\vec{u}}\left(S_{1}\right)$ we have $\sum_{i=1}^{n} r_{i} \delta_{i}=0$ and

$$
T_{\vec{u}}\left(S_{1}\right)=\left\{\vec{\delta}: \delta_{i} \cdot \epsilon_{1}=0, \delta_{n}=0, \sum_{i=1}^{n} r_{i} \delta_{i}=0\right\}
$$

The infinitesimal linear isotropy representation $d \rho$ is given by the linear vector field

$$
F(\delta)=d \rho\left(\frac{\partial}{\partial \theta}\right)(\delta)=\left(\epsilon_{1} \times \delta_{1}, \ldots, \epsilon_{1} \times \delta_{n}\right)
$$

We first compute the Hamiltonian $h$ for $F$ on the larger space $T_{u}(S)$. We claim that

$$
h(\delta)=\frac{1}{2} \sum_{i=1}^{n-1} \eta_{i} r_{i}\left\|\delta_{i}\right\|^{2}
$$

Indeed,

$$
d h(\delta)(\nu)=\sum_{i=1}^{n-1} \eta_{i} r_{i}\left(\delta_{i} \cdot \nu_{i}\right)
$$

for $\nu=\left(\nu_{1}, \ldots, \nu_{n}\right) \in T_{u}(S)$ and

$$
\begin{aligned}
\iota_{F(\delta)} \omega(\nu) & =\sum_{i=1}^{n-1} \eta_{i} r_{i} \epsilon_{1} \cdot\left[\left(\epsilon_{1} \times \delta_{i}\right) \times \nu_{i}\right] \\
& \left.=\sum_{i=1}^{n-1} \eta_{i} r_{i} \epsilon_{1} \cdot\left(\delta_{i} \cdot \nu_{i}\right) \epsilon_{1}\right)
\end{aligned}
$$

and the claim is established. We note that $h$ is a quadratic form of signature $(2 p, 2 q+2)$ (recall that $\eta_{n}>0$ since $e_{n}$ is a forward-track). 
Also, since $S O(2)$ preserves the complex structure $J$, the quadratic form $h$ satisfies $h\left(J \delta, J \delta^{\prime}\right)=h\left(\delta, \delta^{\prime}\right)$, i.e., $h$ is a Hermitian form. Now since $S O(2)$ carries $T_{u}\left(S_{1}\right)$ into itself, $\left.F\right|_{T_{u}\left(S_{1}\right)}$ is tangent to $T_{u}\left(S_{1}\right)$. Hence the restriction of $h$ to $T_{u}\left(S_{1}\right)$, again denoted by $h$, is the Hamiltonian for $\left.F\right|_{T_{u}\left(S_{1}\right)}$. Thus we have only to compute the signature of this restriction. Let $W$ be the orthogonal complement of $T_{u}\left(S_{1}\right)$ in $T_{u}(S)$ for the quadratic form $h$. It is immediate that $W$ is spanned by the two vectors

$$
w_{2}=\left(\eta_{1} \epsilon_{2}, \eta_{2} \epsilon_{2}, \ldots, \eta_{n-1} \epsilon_{2}, 0\right)
$$

and

$$
w_{3}=\left(\eta_{1} \epsilon_{3}, \eta_{2} \epsilon_{3}, \ldots, \eta_{n-1} \epsilon_{3}, 0\right)
$$

Indeed, for $k=2,3$

$$
h\left(w_{k}, \delta\right)=\sum_{i=1}^{n-1} \eta_{i} r_{i} \eta_{i} \epsilon_{k} \cdot \delta_{i}=\sum_{i=1}^{n-1} r_{i} \epsilon_{k} \cdot \delta_{i}=\epsilon_{k} \cdot\left(\sum_{i=1}^{n} r_{i} \delta_{i}\right)
$$

Thus $T_{u}(S)=T_{u}\left(S_{1}\right)+W$ is a direct sum decomposition which is orthogonal for $h$. But $h\left(w_{2}, w_{3}\right)=0$ and $h\left(w_{k}, w_{k}\right)=\sum_{i=1}^{n-1} \eta_{i} r_{i}=-r_{n}<0$, $k=2,3$. Hence $\left.h\right|_{W}$ is negative definite and hence $\left.h\right|_{T_{u}\left(S_{1}\right)}$ is a Hermitian form of signature $(p, q)$.

We now give a complex structure to the symplectic quotient $U / / S O(2)$.

Let $\mathbb{C}^{*}$ act on $\mathbb{C}^{p} \times \mathbb{C}^{q}$ by

$$
\lambda(z, w)=\left(\lambda z, \lambda^{-1} w\right), \lambda \in \mathbb{C}^{*}, z \in \mathbb{C}^{p}, w \in \mathbb{C}^{q}
$$

Let $\left(\mathbb{C}^{p} \times \mathbb{C}^{q}\right)_{s t}$ denote the stable points and $\left(\mathbb{C}^{p} \times \mathbb{C}^{q}\right)_{n s s t}$ denote the nice semi-stable points. Then

$$
\begin{gathered}
\left(\mathbb{C}^{p} \times \mathbb{C}^{q}\right)_{s t}=\left\{(z, w) \in \mathbb{C}^{p} \times \mathbb{C}^{q}: z \neq 0 \text { and } w \neq 0\right\} \\
\left(\mathbb{C}^{p} \times \mathbb{C}^{q}\right)_{n s s t}=\{(0,0)\} \cup\left(\mathbb{C}^{p} \times \mathbb{C}^{q}\right)_{s t}
\end{gathered}
$$

The Mumford quotient $V$ of $\mathbb{C}^{p} \times \mathbb{C}^{q}$ by $\mathbb{C}^{*}$ is by definition the affine variety corresponding to the ring of invariants

$$
\mathbb{C}\left[z_{1}, \ldots, z_{p}, w_{1}, \ldots, w_{q}\right]^{\mathbb{C}^{*}}
$$

It is immediate that this ring is generated by the polynomials $f_{i j}=z_{i} w_{j}$ with relations generated by $f_{i j} f_{j i}=f_{i i} f_{j j}$. Thus $V$ is a homogeneous quadratic cone in $\mathbb{C}^{p q}$. We observe that the topological space $V(\mathbb{C})$ underlying $V$ is the quotient space is the quotient

$$
\left(\mathbb{C}^{p} \times \mathbb{C}^{q}\right)_{n s s t} / \mathbb{C}^{*}
$$

Note that we have an inclusion

$$
\iota: \mathbb{C}^{n-2} / / S O(2) \rightarrow V(\mathbb{C})
$$


The following lemma gives the simplest example relating symplectic quotients with Mumford quotients.

Lemma 2.5. The induced map $\iota$ is a diffeomorphism.

Proof. We will construct an inverse of $\iota$. Let $(z, w) \in\left(\mathbb{C}^{p} \times \mathbb{C}^{q}\right)_{s t}$. Then $\|z\| \cdot\|w\| \neq 0$. Let $\lambda=(\|z\| /\|w\|)^{1 / 2}$. Then $\left(\lambda z, \lambda^{-1} w\right) \in h^{-1}(0)$ since $\|\lambda z\|=\left\|\lambda^{-1} w\right\|$.

By transport of structure we obtain a complex analytic structure on $U / / S O(2)$. This structure clearly agrees with the complex structure already constructed on $U / / S O(2)-\{P\}$.

We have proved the following

Theorem 2.6. $M_{r}$ is a complex analytic space. It has isolated singularities corresponding to the degenerate $n$-gons in $M_{r}$. These singularities are equivalent to homogeneous quadratic cones.

We will now relate the symplectic quotient $\mathcal{M}_{r}$ to the space $Q_{s s t}$. In case the side-lengths $r_{1}, \ldots, r_{n}$ are rational our theorem is a special case of a fundamental theorem of Kirwan, Kempf and Ness [13], [12], [19], relating symplectic quotients with quotients (in the sense of Mumford) of complex projective varieties by complex reductive groups. We note that

$$
r_{1} u_{1}+\ldots+r_{n} u_{n}=0
$$

implies the semi-stability condition. Therefore we have an inclusion $\mu^{-1}(0) \subset M_{n s s t}$ and whence an induced map of quotients

$$
\xi: \mathcal{M}_{r}=\mu^{-1}(0) / S O(3) \rightarrow Q_{s s t}=M_{n s s t} / P S L(2, \mathbb{C})
$$

Theorem 2.7. The map $\xi \circ \epsilon$ is a complex-analytic equivalence.

Proof. In order to prove the theorem we will need some preliminary results on the action of $P S L(2, \mathbb{C})$ on measures on $S^{2}$.

Definition 2.8. A probability measure on $S^{2}$ is called stable if the mass of any atom is less than $1 / 2$. It is called semi-stable if the mass of any atom is not greater than $1 / 2$ and nice semi-stable if it has exactly two atoms each of the mass $1 / 2$.

The following is the basic example of semi-stable measure. Take a vector $\vec{e} \in \mathcal{M}_{r}$, it defines a measure $\nu=\nu(\vec{u}, r)$ on $S^{2}$ by the formula:

$$
\nu=\frac{1}{2} \sum_{j=1}^{n} r_{j} \delta_{u_{j}}
$$

This measure has the total mass 1 and is semi-stable .

Let $i: S^{2} \rightarrow \mathbb{R}^{3}$ be the inclusion. Then the center of mass $B(\nu)$ of a measure $\nu$ on $S^{2}$ is defined by the vector-integral

$$
B(\nu)=\int_{S^{2}} i(u) d \nu(u)
$$


We note that $P S L(2, \mathbb{C})$ acts on measures by push-forward, to be denoted by $\gamma_{*} \nu$ for $\gamma \in P S L(2, \mathbb{C})$ and $\nu$ a measure on $S^{2}$.

Lemma 2.9. For each stable measure $\nu$ on $S^{2}$ there exists $\gamma \in$ $P S L(2, \mathbb{C})$ such that $B\left(\gamma_{*} \nu\right)=0$. The element $\gamma$ is unique up to the postcomposition $g \circ \gamma$ where $g \in S O(3)$.

Proof. Denote by $B^{3}$ the unit ball bounded by $S^{2}$. Douady and Earle in [3] define the conformal center of mass $C(\nu) \in B^{3}$ for any stable probability measure $\nu$ on $S^{2}$. The assignment $C(\nu)$ has the following properties:

(a) For any $\gamma \in P S L(2, \mathbb{C})$

$$
C\left(\gamma_{*} \nu\right)=\gamma(C(\nu))
$$

(b) $B(\nu)=0$ if and only if $C(\nu)=0$.

Note that in (a) the group $P S L(2, \mathbb{C})$ acts on $B^{3}$ as isometries of the hyperbolic 3-space. The lemma follows from the transitivity of this action.

We can now prove that $\xi$ is an isomorphism of complex- analytic spaces. By the previous lemma $\xi \circ \epsilon$ carries the non-singular points of $M_{r}$ continuously to $Q_{s t}$. Also $\xi \circ \epsilon$ carries degenerate $n$-gons to nice semistable points. Thus $\xi \circ \epsilon$ is a continuous bijection and consequently is a homeomorphism. It is easy to check that the complex-linear derivative $d(\xi \circ \epsilon)$ is invertible at all non- singular points. Moreover it follows from the analysis of [4], $\S 4.5$, that $\xi \circ \epsilon$ is a complex- analytic equivalence near the singular points of $M_{r}$.

The theorem follows.

We obtain the following

Corollary 2.10. $M_{r}$ has a natural complex hyperbolic cone structure (see [20] for definitions).

Proof. It is proven in [20] that $M_{n s s t} / P S L(2, \mathbb{C})$ has a complex hyperbolic cone structure.

\section{Bending flows and polygons}

In this section we will show that $M_{r}$ admits actions of $\mathbb{R}^{n-3}$ by bending along $n-3$ "non-intersecting" diagonals (note that $n-3=$ $\left.\frac{1}{2} \operatorname{dim} M_{r}\right)$. The orbits are periodic and there is a dense open subset $M_{r}^{\prime}$ (including the embedded $n$-gons) such that the action can be renormalized to give a Hamiltonian action of the $(n-3)$-torus $\left(S^{1}\right)^{n-3}$ on $M_{r}^{\prime}$. Thus $M_{r}$ is "almost" a toric variety. 
In this paragraph it will be more natural to work with the product

$$
\tilde{M}_{r}=\left\{\vec{e} \in \prod_{j=1}^{n} S^{2}\left(r_{j}\right): \sum_{j=1}^{n} e_{j}=0\right\}
$$

whence $M_{r}=\tilde{M}_{r} / S O(3)$. We need to determine the normalizations of the symplectic forms on factors of $\tilde{M}_{r}$ in order that the zero level set of the associated momentum map $\mu: \tilde{M}_{r} \rightarrow \mathbb{R}^{3}$ is the set of closed $n$-gons. In what follows we will use $\mathcal{S}_{r}$ to be the product $\prod_{j=1}^{n} S^{2}\left(r_{j}\right)$.

Let $\nu$ be the 2 -form on $S^{2}(r)$ given by

$$
\nu_{x}(u, v)=x \cdot(u \times v)=\operatorname{rvol}_{x}(u, v), x \in S^{2}(r), u, v \in T_{x}\left(S^{2}(r)\right)
$$

Here vol denotes the Riemannian volume form on $S^{2}(r)$. We define the symplectic form $\omega$ on $\tilde{M}_{r}$ by

$$
\omega=\sum_{j=1}^{n} \frac{1}{r_{j}^{2}} p_{j}^{*} \nu=\sum_{j=1}^{n} \frac{1}{r_{j}} p_{j}^{*}(v o l)
$$

Lemma 3.1. The momentum map $\mu: \mathcal{S}_{r} \rightarrow \mathbb{R}^{3}$ for the diagonal action of $S O(3)$ on $\left(\mathcal{S}_{r}, \omega\right)$ is given by

$$
\mu(\vec{e})=e_{1}+\ldots+e_{n}
$$

Proof. It suffices to treat the case $n=1$. We replace $r_{1}$ by $r$. Let $w \in \mathbb{R}^{3}=s o(3)$. Then the induced vector field $\hat{w}$ on $S^{2}(r)$ is given by

$$
\hat{w}(x)=w \times x
$$

Let $h_{w}$ be the associated Hamiltonian. It suffices to prove that $h_{w}(x)=$ $w \cdot x$. To this end let $v \in T_{x}\left(S^{2}(r)\right)$. Then $d h_{w}(v)=w \cdot v$ and

$$
\iota_{\hat{w}(x)} \omega_{x}(v)=\frac{1}{r^{2}} x \cdot[(w \times x) \times v]=\frac{1}{r^{2}} x \cdot[(w \cdot v) x]=w \cdot v
$$

Remark 3.2. The equation $\mu(e)=0$ is the "closing condition" for the $n$-gons in $\mathbb{R}^{3}$ with edges $\left(e_{1}, \ldots, e_{n}\right)$. Thus the above normalization for $\omega$ is the correct one. However the map $w \mapsto \hat{w}$ from $\left(\mathbb{R}^{3}, \times\right)$ to the Lie algebra of vector fields on $\mathbb{R}^{3}$ is an antihomomorphism of Lie algebras.

We observe that $\tilde{M}_{r}$ has an $S O(3)$-invariant Kähler structure. The following are the formulas for the Riemannian metric $(\bullet, \bullet)$, symplectic form $\omega$ and almost complex structure $J$ for $\vec{u}, \vec{v} \in T_{\vec{e}} \tilde{M}_{r}$ :

(a) $(\vec{u}, \vec{v})=\sum_{j=1}^{n} \frac{1}{r_{j}} u_{j} \cdot v_{j}$;

(b) $\omega(\vec{u}, \vec{v})=\sum_{j=1}^{n} \frac{e_{j}}{r_{j}^{2}} \cdot\left(u_{j} \times v_{j}\right)$;

(c) $J \vec{u}=\left(\frac{e_{1}}{r_{1}} \times u_{1}, \ldots, \frac{e_{n}}{r_{n}} \times u_{n}\right)$. 
Remark 3.3. The normalization for $\omega$ is chosen in order that $\mu(\vec{e})=$ 0 will be the "closing condition" for $n$-gons. The normalization for $J$ is determined by $J^{2}=-I$. Consequently the normalization for $(\bullet, \bullet)$ is determined as well.

Now let $[P] \in M_{r}$ and choose $\vec{e} \in \tilde{M}_{r}$ corresponding to a closed $n$-gon $P$ in the congruence class $[P]$. We may identify $T_{[P]}\left(M_{r}\right)$ with the orthogonal complement

$$
T_{\vec{e}}^{h o r}\left(\tilde{M}_{r}\right)
$$

of the tangent space to the orbit of $S O(3)$ passing through $\vec{e}$. The subspace $T_{\vec{e}}^{h o r}\left(\tilde{M}_{r}\right)$ consists of vectors $\vec{\delta}=\left(\delta_{1}, \ldots, \delta_{n}\right) \in\left(\mathbb{R}^{3}\right)^{n}$ which satisfy the following equations:

(i) $\delta_{j} \cdot e_{j}=0$;

(ii) $\sum_{j=1}^{n} \delta_{j}=0$;

(iii) $\sum_{j=1}^{n} r_{j}^{-1}\left(e_{j} \times \delta_{j}\right)=0$.

The first equation corresponds to the fixed side lengths of our polygon; the second is the infinitesimal "closing condition" for the polygon $P$. The last equation is the "horizontality" condition due to the following

Remark 3.4. The equation (iii) is equivalent to the condition

(iv) $\sum_{j=1}^{n} r_{j}^{-1}\left(v \times e_{j}\right) \cdot \delta_{j}=0$ for all $v \in \mathbb{R}^{3}$.

We note that the vectors $\left(v \times e_{1}, \ldots, v \times e_{n}\right), v \in \mathbb{R}^{3}$ are the tangents to the $S O(3)$ orbit through $\vec{e}$ in $\tilde{M}_{r}$.

We obtain formulas for the pull-back Riemannian metric $(\bullet, \bullet)$, symplectic form $\omega$ and almost complex structure $J$ on $T_{\vec{e}}^{h o r}\left(\tilde{M}_{r}\right)$ by restricting formulas (a), (b), (c) above. Note that formula (iii) above may be rewritten as

(v) $\sum_{j=1}^{n}(J \delta)_{j}=0$

indicating that $T \underset{\vec{e}}{h o r}\left(\tilde{M}_{r}\right)$ is $J$-invariant.

We now study certain Hamiltonian flows on $M_{r}$. We will identify an $S O(3)$-invariant function on $\left(S^{2}\right)^{n}$ or $\prod_{i=1}^{n} S^{2}\left(r_{i}\right)$ with the function it induces on $M_{r}$ without further comment. We define functions $f_{1}, \ldots, f_{n-3}$ on $\mathcal{S}_{r}$ by

$$
f_{k}\left(e_{1}, \ldots, e_{n}\right)=\frac{1}{2}\left\|e_{1}+\ldots+e_{k+1}\right\|^{2}, k=1, \ldots, n-3
$$

Thus $f_{k}$ corresponds to the length squared of the $k$-th diagonal of $P$ (drawn from $v_{1}$ to $v_{k+2}$ ). Our goal is to find an interpretation of the Hamiltonian flow corresponding to $f_{k}$ in terms of the geometry of $P$.

Lemma 3.5. The Hamiltonian field $H_{f_{k}}$ associated to $f_{k}$ is given by

$$
H_{f_{k}}\left(e_{1}, \ldots, e_{n}\right)=\left(\mu_{k} \times e_{1}, \ldots, \mu_{k} \times e_{k+1}, 0, \ldots, 0\right)
$$


where $\mu_{k}=e_{1}+\ldots+e_{k+1}$ is the $k$-th diagonal of $P$.

Proof. Let $\vec{e}=\left(e_{1}, \ldots, e_{n}\right)$. Since $f_{k}$ does not depend on the last $n-k-1$ components it suffices to prove the lemma for the map

$$
f_{n-1}: \prod_{i=1}^{n} S^{2}\left(r_{i}\right) \rightarrow \mathbb{R}
$$

given by

$$
f_{n-1}(\vec{e})=\frac{1}{2}\left\|e_{1}+\ldots+e_{n}\right\|^{2}=\frac{1}{2}\|\mu\|^{2}
$$

where $\mu$ is the momentum map for the diagonal action of $S O(3)$ on $\prod_{i=1}^{n} S^{2}\left(r_{i}\right)$. By the equivariance of $\mu$ (see the proof of Lemma 3.1 in [13]) the Hamiltonian field $H_{f_{n-1}}$ at $e$ satisfies

$$
H_{f_{n-1}}(\vec{e})=\hat{\mu}(\vec{e})
$$

where $\hat{v}$ is the vector field on $\prod_{i=1}^{n} S^{2}\left(r_{i}\right)$ corresponding to $v \in s o(3)$. But $\hat{v}(\vec{e})=v \times \vec{e}$ and the lemma follows.

Proposition 3.6.

$$
\left\{f_{k}, f_{l}\right\}=0
$$

for all $k, l$.

Proof. We may assume $k<l$. Then

$$
\begin{aligned}
\left\{f_{k}, f_{l}\right\} & =\omega\left(H_{f_{k}}, H_{f_{l}}\right)=\sum_{i=1}^{k+1} \frac{e_{i}}{r_{i}^{2}}\left(\left(\mu_{k} \times e_{i}\right) \times\left(\mu_{l} \times e_{i}\right)\right)= \\
& =\sum_{i=1}^{k+1}\left[e_{i} \cdot\left(\mu_{k} \times \mu_{l}\right)\right]=\mu_{k} \cdot\left(\mu_{k} \times \mu_{l}\right)=0
\end{aligned}
$$

We now study the Hamiltonian flow $\varphi_{k}^{t}$ associated to $f_{k}$. Thus we must solve the system $\left(^{*}\right)$ of ordinary differential equations

$$
\begin{cases}\frac{d e_{i}}{d t} & =\mu_{k} \times e_{i}, 1 \leq i \leq k+1 \\ \frac{d e_{i}}{d t} & =0, k+2 \leq i \leq n\end{cases}
$$

We will use the following notation. Recall that we have identified $\left(\mathbb{R}^{3}, \times\right)$ with the Lie algebra of $S O(3)$ and if $u, v \in \mathbb{R}^{3}$ then we have

$$
\operatorname{ad}_{u}(v)=u \times v
$$

Accordingly we define an element $\exp \left(\operatorname{ad}_{u}\right) \in S O(3)$ as the sum of the power series

$$
\exp \left(\operatorname{ad}_{u}\right)=\sum_{n=0}^{\infty} \frac{\left(\operatorname{ad}_{u}\right)^{n}}{n !}
$$

The following lemma is elementary and is left to the reader.

Lemma 3.7. Let $\Pi$ be the oriented plane in $\mathbb{R}^{3}$ which is orthogonal to $u$. Then $\exp \left(\operatorname{ad}_{u}\right)$ is the rotation in $\Pi$ through an angle of $\|u\|$ radians. 
In particular the curve $\exp \left(t \operatorname{ad}_{u}\right)$ has period $2 \pi /\|u\|$ and angular velocity $\|u\|$.

We can now solve the system $\left({ }^{*}\right)$.

Proposition 3.8. Suppose $P \in M_{r}$ has edges $e_{1}, \ldots, e_{n}$. Then $P(t)=$ $\varphi_{k}^{t}(P)$ has edges $e_{1}(t), . ., e_{n}(t)$ given by

$$
\begin{gathered}
e_{i}(t)=\exp \left(t \operatorname{ad}_{\mu_{k}}\right) e_{i}, 1 \leq i \leq k+1 \\
e_{i}(t)=e_{i}, k+2 \leq i \leq n
\end{gathered}
$$

Proof. We will ignore the last $n-k-1$ edges since they are constants of motion. We make the change of unknown functions

$$
\bar{e}_{1}=e_{1}+\ldots+e_{k+1}=\mu_{k}, \bar{e}_{i}=e_{i}, 2 \leq i \leq k+1
$$

It is immediate that $\bar{e}_{1}, \ldots, \bar{e}_{k+1}$ satisfy the new system of equations:

$$
\begin{gathered}
\frac{d \bar{e}_{1}}{d t}=0 \\
\frac{d \bar{e}_{i}}{d t}=\bar{e}_{1} \times \bar{e}_{i}, 2 \leq i \leq k+1
\end{gathered}
$$

Since $\bar{e}_{1}=\mu_{k}$ we find that $\mu_{k}$ is invariant under the flow and

$$
e_{i}(t)=\exp \left(t \operatorname{ad}_{\mu_{k}}\right) e_{i}, 2 \leq i \leq n
$$

It remains to find $e_{1}(t)$. Note that $\exp \left(t \operatorname{ad}_{\mu_{k}}\right) \mu_{k}=\mu_{k}$, thus

$$
\begin{aligned}
e_{1}(t)=\mu_{k}(t)-\sum_{i=2}^{k+1} e_{i}(t) & =\exp \left(t \operatorname{ad}_{\mu_{k}}\right) \mu_{k}-\sum_{i=2}^{k+1} \exp \left(t \operatorname{ad}_{\mu_{k}}\right) e_{i}= \\
& \exp \left(t \operatorname{ad}_{\mu_{k}}\right) e_{1}
\end{aligned}
$$

Corollary 3.9. The curve $\varphi_{k}^{t}(P)$ is periodic with period $2 \pi / \ell_{k}$ where

$$
\ell_{k}=\left\|e_{1}+\ldots+e_{k+1}\right\|
$$

is the length of the $k$-th diagonal $\mu_{k}$ of $P$.

Remark 3.10. If the $k$-th diagonal has zero length (thus $v_{1}=v_{k+1}$ ) then $P$ is a fixed point of $\varphi_{k}^{t}$. In this case the flow has infinite period.

We see that $\varphi_{k}^{t}(P)$ is the bending flow described in the introduction. It rotates one part of $P$ around the $k$-th diagonal with angular velocity equal to the length of the $k$-th diagonal and leaves the other part fixed.

We next let $M_{r}^{\prime} \subset M_{r}$ be the subset of $M_{r}$ consisting of those $P$ for which no diagonal $\mu_{i}$ has zero length. Then $M_{r}^{\prime}$ is Zariski open in $M_{r}$. 
The functions $\ell_{1}, \ldots, \ell_{n-3}$ are smooth on $M_{r}^{\prime}$ and they Poisson commute. Since $f_{k}=\ell_{k}^{2} / 2$ we have

and consequently

$$
d \ell_{k}=\frac{d f_{k}}{\ell_{k}}
$$

$$
H_{\ell_{k}}=H_{f_{k}} / \ell_{k}
$$

Since $\ell_{k}$ is an invariant of motion the solution procedure in Proposition 3.8 works for $H_{\ell_{k}}$ as well. Let $\Psi_{k}^{t}$ be the flow of $H_{\ell_{k}}$. We obtain the following

Proposition 3.11. Suppose $P \in M_{r}^{\prime}$ has edges $e_{1}, \ldots, e_{n}$. Then $P(t)=\Psi_{k}^{t}(P)$ has edges $e_{1}(t), \ldots, e_{n}(t)$ given by

$$
\begin{gathered}
e_{i}(t)=\exp \left(t \operatorname{ad}_{\mu_{k}} / \ell_{k}\right) e_{i}, 1 \leq i \leq k \\
e_{i}(t)=e_{i}, k+1 \leq i \leq n
\end{gathered}
$$

Thus $\Psi_{k}^{t}$ rotates a part of $P$ around the $k$-th diagonal with constant angular velocity 1 . Hence $\Psi_{k}^{t}(P)$ has period $2 \pi$ and we have proved the following

Theorem 3.12. The space $M_{r}^{\prime}$ of $n$-gons such that no diagonal drawn from the 1-st vertex has zero length, admits a free Hamiltonian action by a torus $T$ of dimension $n-3=\frac{1}{2} \operatorname{dim} M_{r}^{\prime}$.

Remark 3.13. If $n=4,5,6$ then $M_{r}$ is a toric variety for generic $r$ by [2]. For $n=4,5$ it suffices to use the above choice of diagonals. For $n=6$ we have to make different choice of diagonals: $\left[v_{1}, v_{3}\right],\left[v_{3}, v_{5}\right],\left[v_{5}, v_{1}\right]$. Then if $r_{j} \neq r_{i}$ for all $i \neq j$ we conclude that $M_{r}^{\prime}=M_{r}$. Unfortunately, for heptagons any choice of "nonintersecting" diagonals leads to $M_{r}^{\prime} \neq$ $M_{r}$ even for generic values of $r$.

Remark 3.14. In what follows we will also denote by $\Psi_{d}^{t}$ the normalized bending in the diagonal $d$ of the polygon $P$.

\section{Action-angle coordinates}

In this section we use the geometry of $P$ to introduce global actionangle coordinates on the space $M_{r}^{0}$ (which was defined in the Introduction).

In $\S 4,5$ we will use the embedding in $M_{r}$ of the moduli space $N_{r}$ of planar polygons with fixed side lengths modulo the full group of isometries of $\mathbb{E}^{2}$. This embedding is constructed as follows. Let $\Pi$ be a fixed Euclidean plane in $\mathbb{E}^{3}$ and $\sigma$ be the involution of $\mathbb{E}^{3}$ with $\Pi$ as fixed-point set. Then $\sigma$ acts on $M_{r}$. We claim that the fixed-point set of $\sigma$ on $M_{r}$ consists of the polygons that lie in $\Pi$ (up to isometry). Indeed, 
let $P$ be a $n$-gon in $\mathbb{E}^{3}$ which is fixed by $\sigma$ up to a proper isometry. Hence there exists a proper Euclidean motion $g$ such that $\sigma P=g P$. But if the vertices of $P \operatorname{span} \mathbb{E}^{3}$ we have $\sigma=g$, a contradiction. Hence $P$ lies in a plane and can be moved into $\Pi$ by an isometry. The claim follows.

Let $P$ be a $n$-gon in $M_{r}$ and $P^{0}$ be a convex $n$-gon in $\mathbb{R}^{2}$. The diagonals $d=\left[v_{i}, v_{j}\right], d^{\prime}=\left[v_{k}, v_{s}\right]$ of $P$ are called "disjoint" (or "nonintersecting") if the corresponding diagonals of $P^{0}$ do not intersect in the interior of $P^{0}$.

Fix a maximal collection of "disjoint diagonals" $d_{1}, . ., d_{n-3}$ of $P$.

Lemma 4.1. There exists a bending $b$ of $P$ in diagonals $d_{1}, . ., d_{n-3}$ such that $b P$ is a planar polygon.

Proof. The assertion is obvious for quadrilaterals. The general case follows by induction.

Corollary 4.2. The space $M_{r}$ is connected.

Proof. The space $N_{r}$ is connected by [10].

Pick a polygon $P \in M_{r}^{0}$. The diagonals $\mu_{k}, 1 \leq k \leq n-3$ divide $P$ into $n-2$ nondegerate triangles $\Delta_{1}, \ldots, \Delta_{n-2}$ such that $\mu_{k+1}$ is a common side of $\Delta_{k}$ and $\Delta_{k+1}$. We orient $\mu_{k}$ in the direction $v_{k}-v_{1}$. Let $\hat{\theta}_{k}$ be the element of $\mathbb{R} / 2 \pi \mathbb{Z}$ given by the dihedral angle measured from $\Delta_{k}$ to $\Delta_{k+1}, 1 \leq k \leq n-3$ (see Introduction). So $\exp \left(i \hat{\theta}_{k}\right)$ rotates the plane of $\Delta_{k}$ in the positive direction around $\mu_{k}$ into the plane of $\Delta_{k+1}$. Recall that $\theta_{k}=\pi-\hat{\theta}_{k}$.

Lemma 4.3.

$$
\left\{\theta_{i}, \ell_{j}\right\}=\delta_{i j}
$$

Proof. From our description of the bending flows we have

$$
\theta_{i}\left(\Psi_{j}^{t}(P)\right) \equiv \theta_{i}(P)+t \delta_{i j}(\bmod 2 \pi \mathbb{Z})
$$

We obtain the lemma by differentiating.

Corollary 4.4.

$$
\left[H_{\theta_{i}}, H_{\ell_{j}}\right]=0
$$

In order to prove that

$$
\left\{\theta_{1}, \ldots, \theta_{n-3}, \ell_{1}, \ldots, \ell_{n-3}\right\}
$$

are action-angle coordinates it suffices to prove the following

Lemma 4.5.

$$
\left\{\theta_{i}, \theta_{j}\right\}=0
$$


Proof. Recall that $N_{r}$ is the subspace of planar polygons with fixed side lengths modulo the full group of planar Euclidean motions. We have seen that $N_{r}$ is the fixed submanifold of $M_{r}$ under the involution $\sigma$. We note that

$$
\sigma^{*} \theta_{i}=-\theta_{i}, 1 \leq i \leq n-3
$$

Hence $\sigma^{*} d \theta_{i}=-d \theta_{i}$ and since $\sigma^{*} \omega=-\omega$ we have

$$
\sigma_{*} H_{\theta_{i}}=H_{\theta_{i}}, 1 \leq i \leq n-3
$$

Hence if $P$ is a planar polygon we have

$$
H_{\theta_{i}}(P) \in T_{P}\left(N_{r}\right), 1 \leq i \leq n-3
$$

Since $N_{r}$ is Lagrangian we have for $P \in N_{r}$

$$
\omega_{P}\left(H_{\theta_{i}}(P), H_{\theta_{j}}(P)\right)=0,1 \leq i, j \leq n-3
$$

Now let $P$ be a general element of $M_{r}^{0}$. There exists $b \in T$ such that $b P \in$ $N_{r}$ (see Lemma 4.1). Since the $H_{b_{i}}$ and $H_{\theta_{j}}$ commute by Corollary 4.4, the Hamiltonian fields $H_{\theta_{i}}, 1 \leq i \leq n-3$ are invariant under bending and consequently

$$
H_{\theta_{i}}(b P)=d b H_{\theta_{i}}(P), 1 \leq i \leq n-3
$$

Since $\omega$ is invarant under $b$ we have

$$
\omega_{P}\left(H_{\theta_{i}}(P), H_{\theta_{j}}(P)\right)=\omega_{b P}\left(d b\left(H_{\theta_{i}}(P)\right), d b\left(H_{\theta_{j}}(P)\right)=0\right.
$$

The lemma follows.

We have proved the following

Theorem 4.6.

$$
\left\{\theta_{1}, \ldots, \theta_{n-3}, \ell_{1}, \ldots, \ell_{n-3}\right\}
$$

are action-angle coordinates on $M_{r}^{0}$.

\section{The connection with gauge theory and the results of Goldman and Jeffrey-Weitsman}

In this section we first review the description of $M_{r}$ given in [11] in terms of (relative) deformations of flat principal $E(3)$-bundles over the $n$ times punctured 2- sphere $\Sigma$ (here $E(3)$ denotes the group of orientationpreserving isometries of $\mathbb{R}^{3}$ ). We then show that the Lie algebra $e(3)$ of $E(3)$ admits an invariant, non- degenerate symmetric bilinear form $b$ (not the Killing form of course). This form is closely related to the scalar triple product in $\mathbb{R}^{3}$. We use the form $b$ together with wedge product to give a gauge-theoretic description of the symplectic structure on $M_{r}$. This description is the analogue of the usual one in the semisimple case- 
the form $b$ replaces the Killing form. It is then clear how our results on bending are analogues for $E(3)$ (and relative deformations) of those of [7], [9] and [22].

We begin by briefly reviewing our paper [11] on relative deformation theory. It is more convenient to use relative deformations of representations here - for the details of the correspondence with flat connections see $[11]$.

Let $\Gamma$ be a finitely-generated group, $R=\left\{\Gamma_{1}, \ldots, \Gamma_{r}\right\}$ a collection of subgroups of $\Gamma, G$ be the set of real points of an algebraic group defined over $\mathbb{R}$ and $\rho_{0}: \Gamma \rightarrow G$ a representation. In [11] we introduce the relative representation variety $\operatorname{Hom}(\Gamma, R ; G)$. Real points of this variety consist of representations $\rho: \Gamma \rightarrow G$ such that $\left.\rho\right|_{\Gamma_{j}}$ is a representation in the closure of the conjugacy class of $\left.\rho_{0}\right|_{\Gamma_{j}}$. For any linkage $\Lambda$ with $n$ vertices in the Euclidean space $\mathbb{E}^{m}$ we constructed an isomorphism of affine algebraic varieties

$$
\Phi: C(\Lambda) \rightarrow \operatorname{Hom}\left(\Phi_{n}, R ; \hat{E}(m)\right)
$$

Here $C(\Lambda)$ is the configuration space of the linkage $\Lambda$ (we do not divide out by the action of $E(m))$. The group $\Phi_{n}$ is the free product of $n$ copies of $\mathbb{Z} / 2, R$ is a collection of "dihedral" subgroups $\mathbb{Z} / 2 * \mathbb{Z} / 2$ of $\Phi_{n}$ determined by the edges of the linkage and $\hat{E}(m)$. is the full group of isometries of the Euclidean space.

We assume henceforth that the linkage $\Lambda$ is an $n$-gon in $\mathbb{E}^{3}$ with sidelengths $r=\left(r_{1}, \ldots, r_{n}\right)$ and (as above) $M_{r}$ denotes the moduli space $M(\Lambda)=C(\Lambda) / E(3)$. We have an induced isomorphism

$$
\Psi: \operatorname{Hom}\left(\Phi_{n}, R ; \hat{E}(m)\right) / E(m) \rightarrow M(\Lambda)
$$

for any linkage $\Lambda$.

Let $\Sigma=S^{2}-\left\{p_{1}, \ldots, p_{n}\right\}$ denote the 2 -sphere punctured at $\left\{p_{1}, \ldots, p_{n}\right\}$, let $U_{1}, \ldots, U_{n}$ denote disjoint disc neighborhoods of $p_{1}, \ldots, p_{n}$ and $U=$ $U_{1} \cup \ldots \cup U_{n}$. The subgroup $\Gamma_{n} \subset \Phi_{n}$ consisting of words of even length in the generators $\tau_{1}, \ldots, \tau_{n}$ is isomorphic to $\pi_{1}(\Sigma)$ (see [11], Lemma 4.1). Indeed, put $\gamma_{i}=\tau_{i} \tau_{i+1}, 1 \leq i \leq n$. Then $\gamma_{1} \cdot \ldots \cdot \gamma_{n}=1$. Let $\rho \in$ $\operatorname{Hom}\left(\Phi_{n}, R ; \hat{E}(m)\right)$. Then $\rho$ induces a representation $\rho: \Gamma_{n} \rightarrow E(3)$ and a flat principal $E(3)$-bundle $P$ over $\Sigma$. We let ad $P$ be the associated flat Lie algebra bundle. In our case we can use the restriction map to replace the above relative representation variety with one that makes the connection with $M_{r}$ transparent. Let $T$ be the set of conjugacy classes in $\Gamma_{n}$ given by

$$
T=\left\{C\left(\gamma_{1}\right), \ldots, C\left(\gamma_{n}\right)\right\}
$$


Here $C(\gamma)$ denotes the conjugacy class of $\gamma$. Then it is immediate that we have an induced isomorphism

$$
\Phi: \operatorname{Hom}\left(\Gamma_{n}, T ; E(3)\right) / E(3) \rightarrow M_{r}
$$

Indeed, each $n$-gon corresponds to the $n$ translations in the directions of its edges $e_{1}, \ldots, e_{n}$. The relation $\gamma_{1} \cdot \ldots \cdot \gamma_{n}=1$ corresponds to the closing condition

$$
e_{1}+\ldots+e_{n}=0
$$

Note that $r_{i}$ is the translational length of $\rho_{0}\left(\gamma_{i}\right)$. We will henceforth abbreviate $\operatorname{Hom}\left(\Gamma_{n}, T ; E(3)\right) / E(3)$ to $X_{n, r}$.

Remark 5.1. If $\alpha$ is an automorphism of $\Gamma_{n}$ that preserves each class

$$
C\left(\gamma_{1}\right), \ldots, C\left(\gamma_{n}\right)
$$

then $\alpha$ acts trivially on $\operatorname{Hom}\left(\Gamma_{n}, T ; E(3)\right)$. Indeed, since $\rho\left(\Gamma_{n}\right)$ is contained in the translation subgroup of $E(3)$, fixing the conjugacy class of $\rho(\gamma)$ amounts to fixing $\rho(\gamma)$. Thus quantizing $M_{r}$ will produce only trivial representations of the pure braid group.

Let $\rho \in X_{n, r}$. We define the parabolic cohomology

$$
H_{p a r}^{1}(\Sigma, \operatorname{ad} P)
$$

to be the subspace of the de Rham cohomology classes in $H^{1}(\Sigma$, ad $P)$ whose restrictions to each $U_{i}$ are trivial. By [11], we may calculate the relative deformations of $\rho$ and consequently $H_{\text {par }}^{1}(\Sigma, \operatorname{ad} P)$ by using the differential graded Lie algebra $\mathcal{B}^{\bullet}(\Sigma, U ;$ ad $P)$. This algebra is the subalgebra of the de Rham algebra consisting of sections of ad $P$ which are constant on $U$ in degree zero and ad $P$ - valued forms which vanish on $U$ in degrees 1 and 2.

We now give our gauge-theoretic description of the symplectic form on $M_{r}$. Since the Lie algebra $e(3)$ of $E(3)$ is not semi-simple, the Killing form of $e(3)$ is degenerate and we can not give the usual (i.e., for $G$ semisimple) description of the symplectic form. However it is a remarkable fact that there is another $E(3)$-invariant symmetric form $b$ on $e(3)$ which we now describe. We recall that we may identify $\Lambda^{2} \mathbb{R}^{3}$ with $s o(3)$ by associating to $u \wedge v$ the element of $\operatorname{End}\left(\mathbb{R}^{3}\right)$ (also denoted by $u \wedge v$ ) given by

$$
(u \wedge v)(w)=(u, w) v-(v, w) u
$$

We define a bilinear form

$$
a: s o(3) \times \mathbb{R}^{3} \rightarrow \mathbb{R}
$$

by

$$
a(u \wedge v, w)=(u \times v) \cdot w
$$


We split $e(3)$ according to $e(3)=s o(3)+\mathbb{R}^{3}$ and define the (split) form $b: e(3) \times e(3) \rightarrow \mathbb{R}$ by

$$
b\left(\left(u_{1} \wedge v_{1}, w_{1}\right),\left(u_{2} \wedge v_{2}, w_{2}\right)\right)=a\left(u_{1} \wedge v_{1}, w_{2}\right)+a\left(u_{2} \wedge v_{2}, w_{1}\right)
$$

The following proposition is immediate

Proposition 5.2. The form $b$ is symmetric, nondegenerate and invariant under $E(3)$.

We combine the form $b$ on $e(3)$ with the wedge-product to obtain a skew-symmetric bilinear form

$$
B: H_{p a r}^{1}(\Sigma, \operatorname{ad} P) \times H_{p a r}^{1}(\Sigma, \operatorname{ad} P) \rightarrow H^{2}(\Sigma, U ; \mathbb{R})
$$

we then evaluate on the relative fundamental class of $M$ to obtain a skew symmetric form

$$
A: H_{\text {par }}^{1}(\Sigma, \operatorname{ad} P) \times H_{p a r}^{1}(\Sigma, \operatorname{ad} P) \rightarrow \mathbb{R}
$$

It follows from Poincare duality that $A$ is nondegenerate and we obtain a symplectic structure on $X_{n, r}$. In order to relate $A$ to the symplectic form $\omega$ of Section we need to make explicit the induced isomorphism

$$
d \Psi_{p}: T_{\rho}\left(X_{n, r}\right) \rightarrow T_{\rho}\left(M_{r}\right)
$$

To do this we need to pass through the group cohomology description of $H_{\text {par }}^{1}(\Sigma, \operatorname{ad} P)$. In the following discusssion we let $G$ be any Lie group. We denote by $\mathcal{G}$ the Lie algebra of $G$. Recall that we may identify the universal cover $\tilde{\Sigma}$ of $\Sigma$ with the hyperbolic plane $\mathbb{H}^{2}$ - we will make this explicit later. Let $p: \tilde{\Sigma} \rightarrow \Sigma$ be the covering projection. We will identify $\mathcal{A}^{\bullet}\left(\tilde{\Sigma}, p^{*}\right.$ ad $P$ ) with the $\mathcal{G}$-valued differential forms on $\tilde{\Sigma}$ (via parallel translation from a point $\left.v_{1}\right)$. Given $[\eta] \in H^{1}(\Sigma$, ad $P)$ choose a representing closed 1 -form $\eta \in \mathcal{A}^{\bullet}(\Sigma$, ad $P)$. Let $\tilde{\eta}=p^{*} \eta$ and $f: \tilde{\Sigma} \rightarrow \mathcal{G}$ be the unique function satisfying:

$$
\begin{aligned}
& \text { - } f\left(v_{1}\right)=0 \\
& \text { - } d f=\tilde{\eta} \text {. }
\end{aligned}
$$

We define a 1-cochain $h(\eta) \in Z^{1}(\Gamma, \mathcal{G})$ with coefficients in $\mathcal{G}$ by

$$
h(\eta)(\gamma)=f(x)-\operatorname{Ad} \rho(\gamma) f\left(\gamma^{-1} x\right)
$$

We note that the right-hand side does not depend on $x$. We define $\tau([\eta])$ to be the class of $h(\eta)$ in $H^{1}(\Gamma, \mathcal{G})$. It is easily checked (see [8], §4) that $\tau$ is an isomorphism. We note that $[\eta] \in H_{p a r}^{1}(M$, ad $P)$ if and only if the restriction of $h(\eta)$ to the cyclic groups generated by $\gamma_{i}$ are exact for all $i$. We denote the set of all 1-cocycles in $Z^{1}(\Gamma, \mathcal{G})$ satisfying this property by $Z_{\text {par }}^{1}(\Gamma, \mathcal{G})$. 
We now return to the case $G=E(3)$. Let $\rho \in \operatorname{Hom}\left(\Gamma_{n}, T ; E(3)\right)$. Since $\rho_{0}(\gamma)$ is a translation for all $\gamma \in \Gamma_{n}$ it follows that if

$$
c \in T_{\rho}\left(\operatorname{Hom}\left(\Gamma_{n}, T ; E(3)\right)\right.
$$

then $c(\gamma)$ is an infinitesimal translation for all $\gamma \in \Gamma$ and consequently we may identify $c$ with an element of $\operatorname{Hom}\left(\Gamma_{n}, \mathbb{R}^{3}\right)$. The condition that $c$ is a parabolic cocycle is equivalent to $c\left(\gamma_{i}\right) \cdot e_{i}=0$ where $\rho\left(\gamma_{i}\right)$ is a translation by $e_{i}$. We leave the proof of the next lemma to the reader.

Lemma 5.3.

$$
d \Psi_{\rho}(c)=\vec{\delta}=\left(\delta_{1}, \ldots, \delta_{n}\right) \in T_{\vec{e}}\left(M_{r}\right)
$$

where $\delta_{i}=c\left(\gamma_{i}\right), 1 \leq i \leq n$.

Remark 5.4. Here we think of $T_{\vec{e}}\left(M_{r}\right)$ as the quotient of $T_{\vec{e}}\left(\tilde{M}_{r}\right)$ by $S O(3)$, see $\S 3$ for the definitions.

We can now state the main result of the next section. Recall that the symplectic structure $\omega$ on $M_{r}$ was described in $\S 3$.

Theorem 5.5. With the above identification we have $A=\omega$.

Proof. We will work in the more general framework where $E(3)$ is replaced by a Lie group $G$ admitting an invariant symmetric bilinear form on its Lie algebra $\mathcal{G}$. We have in mind an eventual application to $n$-gon linkages in $S^{3}$.

We construct a fundamental domain $D$ for $\Gamma_{n}$ operating in $\mathbb{H}^{2}$ as follows. Choose a point $x_{0}$ on $\Sigma$ and make cuts along geodesics from $x_{0}$ to the cusps. The resulting fundamental domain $D$ is a geodesic $2 n$-gon with $n$ interior vertices $v_{1}, \ldots, v_{n}$ and $n$ cusps $v_{1}^{\infty}, \ldots, v_{n}^{\infty}$. These occur alternately so that proceeding counterclockwise around $\partial D$ we see $v_{1}, v_{1}^{\infty}, v_{2}, v_{2}^{\infty}, \ldots, v_{n}, v_{n}^{\infty}$. The generator $\gamma_{i}$ fixes $v_{i}^{\infty}$ and satisfies $\gamma_{i}\left(v_{i}\right)=$ $v_{i+1}$. We take $v_{1}$ as our base point $x_{0}$ in $\mathbb{H}^{2}$.

Remark 5.4. We have changed our original generators of $\Gamma_{n}$ to their inverses.

Now let $\rho \in \operatorname{Hom}\left(\Gamma_{n}, T ; G\right) / G$ and $c, c^{\prime} \in H_{p a r}^{1}\left(\Gamma_{n}, \mathcal{G}\right)$ be tangent vectors at $\rho$. Let $\alpha$ and $\alpha^{\prime}$ be the corresponding elements of the de Rham cohomology group $H_{p a r}^{1}(\Sigma$, ad $P)$. By assumption there exist vectors $w_{i}, w_{i}^{\prime} \in \mathcal{G}, 1 \leq i \leq n$, such that

$$
\begin{aligned}
w_{i}-\operatorname{Ad} \rho\left(\gamma_{i}\right) w_{i} & =c\left(\gamma_{i}\right) \\
w_{i}^{\prime}-\operatorname{Ad} \rho\left(\gamma_{i}\right) w_{i}^{\prime} & =c^{\prime}\left(\gamma_{i}\right)
\end{aligned}
$$

We let $B .(\Gamma)$ be the bar resolution of $\Gamma$, [15]. Thus $B_{k}(\Gamma)$ is the free $\mathbb{Z}[\Gamma]$-module on the symbols $\left[\gamma_{1}\left|\gamma_{2}\right| \ldots \mid \gamma_{k}\right]$ with

$$
\partial\left[\gamma_{1}\left|\gamma_{2}\right| \ldots \mid \gamma_{k}\right]=\gamma_{1}\left[\gamma_{2}|\ldots| \gamma_{k}\right]+
$$




$$
\sum_{i=1}^{k-1}\left[\gamma_{1}|\ldots| \gamma_{i} \gamma_{i+1}|\ldots| \gamma_{k}\right]+(-1)^{k}\left[\gamma_{1}\left|\gamma_{2}\right| \ldots \mid \gamma_{k-1}\right]
$$

We let $C_{k}(\Gamma)=B_{k}(\Gamma) \otimes_{\mathbb{Z}[\Gamma]} \mathbb{Z}$, where $\mathbb{Z}[\Gamma]$ acts on $\mathbb{Z}$ via the homomorphism $\epsilon$ defined by

$$
\epsilon\left(\sum_{i=1}^{m} a_{i} \gamma_{i}\right)=\sum_{i=1}^{m} a_{i}
$$

Thus $C_{k}(\Gamma)$ is the free abelian group on the symbols $\left(\gamma_{1}|\ldots| \gamma_{k}\right)=\left[\gamma_{1}\left|\gamma_{2}\right| \ldots \mid \gamma_{k}\right] \otimes 1$ and

$$
\begin{gathered}
\partial\left(\gamma_{1}|\ldots| \gamma_{k}\right)=\left(\gamma_{2}|\ldots| \gamma_{k}\right)+ \\
\sum_{i=1}^{k-1}\left(\gamma_{1}|\ldots| \gamma_{i} \gamma_{i+1}|\ldots| \gamma_{k}\right)+(-1)^{k}\left(\gamma_{1}\left|\gamma_{2}\right| \ldots \mid \gamma_{k-1}\right)
\end{gathered}
$$

We define a relative fundamental class $F \in C_{2}(\Gamma)$ by the property:

$$
\partial F=\sum_{i=1}^{n}\left(\gamma_{i}\right)
$$

Let $[\Gamma, \partial \Gamma] \in C_{2}(\Gamma)$ be the chain

$$
[\Gamma, \partial \Gamma]=\sum_{i=2}^{n}\left(\gamma_{i} \mid \gamma_{i-1} \gamma_{i-2} \ldots \gamma_{1}\right)
$$

The reader will easily verify the following lemma which was pointed out to us by Valentino Zocca.

Lemma 5.7. $[\Gamma, \partial \Gamma]$ is a relative fundamental class.

We abuse notation and use $B(\cdot, \cdot)$ to denote the above wedge product of the de Rham cohomology classes and the cup-product of EilenbergMacLane cochains using the form $b$ on the coefficients.

\section{Proposition 5.8.}

$$
\int_{\Sigma} B\left(\alpha, \alpha^{\prime}\right)=\left\langle B\left(c, c^{\prime}\right),[\Gamma, \partial \Gamma]\right\rangle-\sum_{i=1}^{n}\left\langle B\left(c, w_{i}^{\prime}\right),\left(\gamma_{i}\right)\right\rangle
$$

Proof. The reader will verify that the right-hand side of this formula does not depend on the choices of $w_{i}^{\prime}, 1 \leq i \leq n$ and of a relative fundamental class $[\Gamma, \partial \Gamma]$. In the following we let $e_{i}$ be the oriented edge of $\partial D$ joining $v_{i}$ to $v_{i}^{\infty}$ and $\check{e}_{i}$ be the oriented edge joining $v_{i}^{\infty}$ to $v_{i+1}$. Then $\gamma_{i} e_{i}=-\check{e}_{i}$. We remind the reader that the 1 -forms $\alpha$ and $\alpha^{\prime}$ vanish in neighborhoods of the cusps $v_{i}^{\infty}, 1 \leq i \leq n$. Proposition 5.8 will be a consequence of the following three lemmas. 


\section{Lemma 5.9.}

$$
\int_{e_{i}} B\left(f, \alpha^{\prime}\right)+\int_{\tilde{e}_{i}} B\left(f, \alpha^{\prime}\right)=b\left(c\left(\gamma_{i}\right), f^{\prime}\left(v_{i+1}\right)\right)-b\left(c\left(\gamma_{i}\right), v_{i}^{\infty}\right)
$$

Proof.

$$
\begin{aligned}
\int_{e_{i}} B\left(f, \alpha^{\prime}\right) & +\int_{\check{e}_{i}} B\left(f, \alpha^{\prime}\right) \\
= & \int_{\tilde{e}_{i}} B\left(f, \alpha^{\prime}\right)-\int_{\gamma_{i}^{-1} \tilde{e}_{i}} B\left(f, \alpha^{\prime}\right) \\
= & \int_{\tilde{e}_{i}}\left[B\left(f, \alpha^{\prime}\right)-\left(\gamma_{i}^{-1}\right)^{*} B\left(f, \alpha^{\prime}\right)\right] \\
= & \int_{\tilde{e}_{i}}\left[B\left(f, \alpha^{\prime}\right)-B\left(\left(\gamma_{i}^{-1}\right)^{*} f,\left(\gamma_{i}^{-1}\right)^{*} \alpha^{\prime}\right)\right] \\
= & \int_{\tilde{e}_{i}}\left[B\left(f, \alpha^{\prime}\right)-B\left(\operatorname{Ad} \rho\left(\gamma_{i}\right)\left(\gamma_{i}^{-1}\right)^{*} f, \operatorname{Ad} \rho\left(\gamma_{i}\right)\left(\gamma_{i}^{-1}\right)^{*} \alpha^{\prime}\right)\right] \\
& =\int_{\tilde{e}_{i}} B\left(f-\operatorname{Ad} \rho\left(\gamma_{i}\right)\left(\gamma_{i}^{-1}\right)^{*} f, \alpha^{\prime}\right)=\int_{\check{e}_{i}} B\left(c\left(\gamma_{i}\right), \alpha^{\prime}\right) \\
& =B\left(c\left(\gamma_{i}\right), f^{\prime}\left(v_{i+1}\right)\right)-B\left(c\left(\gamma_{i}\right), f^{\prime}\left(v_{i}^{\infty}\right)\right)
\end{aligned}
$$

We obtain

$$
\int_{\Sigma} B\left(\alpha, \alpha^{\prime}\right)=\sum_{i=1}^{n} b\left(c\left(\gamma_{i}\right), f^{\prime}\left(v_{i+1}\right)-\sum_{i=1}^{n} b\left(c\left(\gamma_{i}\right), f^{\prime}\left(v_{i}^{\infty}\right)\right)\right.
$$

To evaluate the second sum we need

Lemma 5.10.

$$
\delta f^{\prime}\left(v_{i}^{\infty}\right)\left(\gamma_{i}\right)=c^{\prime}\left(\gamma_{i}\right), 1 \leq i \leq n
$$

were $\delta$ is the Eilenberg-MacLane coboundary.

Proof. By definition for any $x \in \mathbb{H}^{2}$ we have:

$$
c^{\prime}\left(\gamma_{i}\right)=f^{\prime}(x)-\operatorname{Ad}\left(\rho\left(\gamma_{i}\right) f^{\prime}\left(\gamma_{i}^{-1} x\right)\right.
$$

Since $f^{\prime}$ is a covariant constant near the cusps we may allow $x$ to tend to $v_{i}^{\infty}$ in the above formula. Since $\gamma_{i}^{-1} v_{i}^{\infty}=v_{i}^{\infty}$ we obtain

$$
c^{\prime}\left(\gamma_{i}\right)=f^{\prime}\left(v_{i}^{\infty}\right)-\operatorname{Ad}\left(\rho\left(\gamma_{i}\right) f^{\prime}\left(v_{i}^{\infty}\right)\right.
$$

We now evaluate the sum over the interior vertices.

\section{Lemma 5.11.}

$$
\sum_{i=1}^{n} b\left(c\left(\gamma_{i}\right), f^{\prime}\left(v_{i+1}\right)\right)=\left\langle B\left(c, c^{\prime}\right),[\Gamma, \partial \Gamma]\right\rangle
$$




$$
+\sum_{i=1}^{n} b\left(c\left(\gamma_{i}\right), f^{\prime}\left(v_{i}^{\infty}\right)\right)-\sum_{i=1}^{n}\left\langle B\left(c, w_{i}^{\prime}\right),\left(\gamma_{i}\right)\right\rangle
$$

Proof. By definition, for any $x \in \mathbb{H}^{2}, \gamma \in \Gamma$, we have

$$
c^{\prime}(\gamma)=f^{\prime}(x)-\operatorname{Ad} \rho(\gamma) f^{\prime}\left(\gamma^{-1} x\right)
$$

Substituting $x=v_{i+1}, \gamma=\gamma_{i}$ and using $\gamma_{i}^{-1} v_{i+1}=v_{i}$ we obtain

$$
c^{\prime}\left(\gamma_{i}\right)=f^{\prime}\left(v_{i+1}\right)-\operatorname{Ad} \rho\left(\gamma_{i}\right) f^{\prime}\left(v_{i}\right)
$$

Using $f^{\prime}\left(v_{1}\right)=0$ we conclude that

$$
f^{\prime}\left(v_{i}\right)=c\left(\gamma_{i-1} \gamma_{i-2} \ldots \gamma_{1}\right)
$$

Hence

$$
\begin{aligned}
\sum_{i=1}^{n} b\left(c\left(\gamma_{i}\right), f^{\prime}\left(v_{i+1}\right)\right)= & \sum_{i=1}^{n} b\left(c\left(\gamma_{i}\right), c^{\prime}\left(\gamma_{i} \gamma_{i-1} \ldots \gamma_{1}\right)\right) \\
= & \left.\sum_{i=1}^{n} b\left(c\left(\gamma_{i}\right)\right), c^{\prime}\left(\gamma_{i}\right)\right) \\
& +\sum_{i=2}^{n} b\left(c\left(\gamma_{i}\right), \operatorname{Ad} \rho\left(\gamma_{i}\right) c^{\prime}\left(\gamma_{i-1} \ldots \gamma_{1}\right)\right) \\
= & \sum_{i=1}^{n} b\left(c\left(\gamma_{i}\right), c^{\prime}\left(\gamma_{i}\right)\right)+\left\langle B\left(c, c^{\prime}\right),[\Gamma, \partial \Gamma]\right\rangle
\end{aligned}
$$

We substitute $c^{\prime}\left(\gamma_{i}\right)=f^{\prime}\left(v_{i}^{\infty}\right)-\operatorname{Ad} \rho\left(\gamma_{i}\right) f^{\prime}\left(v_{i}^{\infty}\right)$ and use the formula

$$
\left\langle B\left(c, w_{i}^{\prime}\right),\left(\gamma_{i}\right)\right\rangle=b\left(c\left(\gamma_{i}\right), \operatorname{Ad} \rho\left(\gamma_{i}\right) w_{i}^{\prime}\right)
$$

to obtain the lemma.

We have proved Proposition 5.8 and now specialize to the case at hand, namely $G=E(3)$. In this case $c$ and $c^{\prime}$ take values in the Lie subalgebra of infinitesimal translations. Since this is a totally-isotropic subspace for $b$ we obtain

$$
\left\langle B\left(c, c^{\prime}\right),[\Gamma, \partial \Gamma]\right\rangle=0
$$

It remains to evaluate the sum over the cusps.

Lemma 5.12.

$$
\left\langle B\left(c, w_{i}^{\prime}\right),\left(\gamma_{i}\right)\right\rangle=\delta_{i} \cdot\left(\frac{e_{i}}{r_{i}^{2}} \times \delta_{i}^{\prime}\right)
$$

where $c\left(\gamma_{i}\right)=\delta_{i}, c^{\prime}\left(\gamma_{i}\right)=\delta_{i}^{\prime}, 1 \leq i \leq n$ 
Proof. We first note that

$$
\begin{aligned}
\left\langle B\left(c, w_{i}^{\prime}\right),\left(\gamma_{i}\right)\right\rangle & =b\left(c\left(\gamma_{i}\right), \operatorname{Ad} \rho\left(\gamma_{i}\right) w_{i}^{\prime}\right) \\
& =b\left(\operatorname{Ad} \rho\left(\gamma_{i}^{-1}\right) c\left(\gamma_{i}\right), w_{i}^{\prime}\right)=b\left(c\left(\gamma_{i}\right), w_{i}^{\prime}\right)
\end{aligned}
$$

The last equality holds because $\rho\left(\gamma_{i}\right)$ is a translation and $c\left(\gamma_{i}\right)$ is an infinitesimal translation. A direct computation in the Lie algebra $e(3)$ shows that

$$
\frac{e_{i}}{r_{i}^{2}} \wedge \delta_{i}^{\prime}-\operatorname{Ad} \rho\left(\gamma_{i}\right) \frac{e_{i}}{r_{i}^{2}} \wedge \delta_{i}^{\prime}=\delta_{i}^{\prime}
$$

Hence we may choose $w_{i}^{\prime}=\frac{e_{i}}{r_{i}^{2}} \wedge \delta_{i}^{\prime}, 1 \leq i \leq n$, and the lemma follows from the definition of $b$.

We have accordingly

$$
A\left(\alpha, \alpha^{\prime}\right)=\int_{\Sigma} B\left(\alpha, \alpha^{\prime}\right)=-\sum_{i=1}^{n} \delta_{i} \cdot\left(\frac{e_{i}}{r_{i}^{2}} \times \delta_{i}^{\prime}\right)
$$

Comparing with our formula for the symplectic structure in $\S 3$ we obtain Theorem 5.5.

We can now relate our results on the bending of $n$-gon linkages with the work of Goldman, Jeffrey-Weitsman and Weitsman. There are two independent class functions on $E(3)$ : translation length $\ell$ and the trace of the rotation part $t$. We replace $\ell$ by $f=\ell^{2} / 2$ to get a polynomial invariant. Given $\gamma \in \Gamma_{n}$ we define

$$
f_{\gamma}: \operatorname{Hom}\left(\Gamma_{n}, T ; E(3)\right) / E(3) \rightarrow \mathbb{R}
$$

by $f_{\gamma}=f(\rho(\gamma))$. In the case $\gamma=\mu_{i}=\gamma_{1} \gamma_{2} \ldots \gamma_{i+1}, 1 \leq i \leq n-3$ it is easily seen that the Hamiltonian flow of $f_{\gamma}$ corresponds to the (unnormalized) bending flow in the $i$-th diagonal. The decomposition of the polygon $P$ by diagonals drawn from a common vertex corresponds to a decomposition of $\Sigma$ into pairs of pants using the curves $\mu_{1}, \ldots, \mu_{n-3}$. Thus our real polarization of $M_{r}$ (i.e., singular Lagrangian foliation) obtained by bending in the above diagonals corresponds to that of [9] and [22] obtained from "twists" with respect to $\mu_{1}, \ldots, \mu_{n-3}$.

\section{Transitivity of bending deformations}

Definition 6.1. An embedded polygon $P \in M_{r}$ is called a "pseudotriangle" if the union of edges of $P$ is a triangle in $\mathbb{R}^{3}$. The vertices of this triangle are called the "pseudovertices" of the pseudotriangle $P$.

It is easy to see that $M_{r}$ contains only a finite number of "pseudotriangles" $T_{1}, \ldots T_{N}$, where $N<n^{3}$. The main result of this paragraph is the following: 
Theorem 6.2. (a) For each nonsingular moduli space $M_{r}$ there exists a number $\ell=\ell(r)$ such that each polygon $P \in M_{r}$ can be deformed to a pseudotriangle via not more than $\ell$ bendings.

(b) The function $\ell(r)$ is bounded on compacts in $\mathcal{D}_{n}-\Sigma$.

(c) The subset of polygons $P \in M_{r}$ which can be deformed to a pseudotriangle $T$ by at most $\ell$ bendings is closed in $M_{r}$.

(d) Each pair of polygons $P, Q \in M_{r}$ can be deformed to each other by a sequence of not more than $n^{3} \ell$ bendings.

\subsection{Bending of quadrilaterals}

We first consider a special case of bending assuming that:

(a) $P$ is a planar quadrilateral with a nonsingular moduli space $M_{r}$;

(b) we allow sequences of bendings in both diagonals of $P$;

(c) bending angles are always $\pi$ (so the bending of a planar polygon is again a planar polygon). Such a bending will be called a $\pi$-bending.

We have an action of the group $\mathbb{Z}_{2} * \mathbb{Z}_{2}$ on the moduli space $N_{r}$ of planar quadrilaterals given by $\pi$-bendings along the diagonals.

Denote the $\pi$-bending in the diagonal $\left[v_{1}, v_{3}\right]$ by $\alpha$ and the $\pi$-bending in the diagonal $\left[v_{2}, v_{4}\right]$ by $\beta$. We assume that $\alpha$ fixes the vertex $v_{2}$ and $\beta$ fixes the vertex $v_{1}$. We shall normalize 4-gons $P$ so that $e_{1}=r_{1} u_{1}$, $u_{1}=1 \in \mathbb{C}$.

Then

$$
\begin{aligned}
& \alpha:\left(u_{1}, u_{2}, u_{3}, u_{4}\right) \mapsto\left(u_{1}, u_{2}, u_{3}^{-1} \frac{r_{1}+r_{2} u_{2}}{r_{1}+r_{2} u_{2}^{-1}}, u_{4}^{-1} \frac{r_{1}+r_{2} u_{2}}{r_{1}+r_{2} u_{2}^{-1}}\right) \\
& \beta:\left(u_{1}, u_{2}, u_{3}, u_{4}\right) \mapsto\left(u_{1}, u_{2}^{-1} \frac{r_{1}+r_{2} u_{2}}{r_{1}+r_{2} u_{2}^{-1}}, u_{3}^{-1} \frac{r_{1}+r_{2} u_{2}}{r_{1}+r_{2} u_{2}^{-1}}, u_{4}\right)
\end{aligned}
$$

Both maps are birational transformations of $\mathbb{C}^{4}$. The moduli space $N_{r} \cong S^{1}$ is the quotient of the curve

$$
E=\left\{\left(u_{1}, u_{2}, u_{3}, u_{4}\right) \in \mathbb{C}^{4}: u_{1}=1,\left|u_{j}\right|=1, j=2,3,4 ; \sum_{j=1}^{4} r_{j} u_{j}=0\right\}
$$

by action of the involution $\tau:\left(u_{1}, u_{2}, u_{3}, u_{4}\right) \mapsto\left(\bar{u}_{1}, \bar{u}_{2}, \bar{u}_{3}, \bar{u}_{4}\right)$.

Lemma 6.3. The complexification $E^{c}$ of the curve $E$ is a nonsingular connected elliptic curve. The composition $\beta \circ \alpha=\theta$ extends to an automorphism $\theta^{c}$ of $E^{c}$ which is fixed-point free.

Proof. The first statement of the lemma was proven in [5], [6]. The biholomorphic extension $\theta^{c}$ exists since $E$ is Zariski dense in $E^{c}$. The transformation $\theta: E \rightarrow E$ has no fixed points. It follows from the classification of automorphisms of elliptic curves that $\theta^{c}$ is also fixedpoint free. 
In particular the self-map $\theta$ of $E$ preserves the metric on $E$ given by the restriction of a flat metric on $E^{c}$. Therefore if we identify $E /\langle\tau\rangle$ with the unit circle then $\theta: S^{1} \rightarrow S^{1}$ is a rotation. Denote by $a_{s}, b_{t}$ the 1 -parameter families of bendings in the diagonals $\left[v_{1}, v_{3}\right],\left[v_{2}, v_{4}\right]$ so that $a_{\pi}=\alpha, b_{\pi}=\beta$.

Lemma 6.4. An arc $[x, \beta \circ \alpha(x)]$ between $x, \theta(x)$ on $S^{1}$ is contained in the orbit $b_{t(s)} \circ a_{s}(x)$, where $s, t \in \mathbb{R} / 2 \pi \mathbb{Z}$.

Proof. For each point $a_{s}(x)$ we take $b_{t(s)}$ to be one of two bendings which makes $a_{s}(x)$ planar. We choose $b_{t(s)}$ so that it depends continuously on $s$ and $b_{t(0)}=b_{0}=i d, b_{t(\pi)}=b_{\pi}=\beta$. It is clear that for $s=\pi$ the polygon $b_{t(s)} \circ a_{s}(x)$ is equal to $\theta(x)$. This proves the Lemma.

Corollary 6.5. Suppose that $r$ does not belong to a face of the polyhedron $\mathcal{D}_{4}-\Sigma$. Let $\gamma$ be the rotation angle of the element $\theta=$ $\beta \circ \alpha, m=[2 \pi / \gamma]+1$. Then for each two points $x, y$ in the space of quadrilaterals $M_{r}$ there exists a composition of at most $2 m+2$ bendings which transforms $x$ to $y$.

Proof. Our assumptions imply that the moduli space $M_{r}$ is not a single point. Thus the angle $\gamma$ is different from zero. We first apply a single bending to each $x, y$ to make them planar polygons $x^{\prime}, y^{\prime}$. There exists a composition of at most $2 m-2 \pi$-bendings which sends $x^{\prime}$ to a point $x^{\prime \prime}$ on the $\operatorname{arc}\left[y^{\prime}, \alpha \circ \beta\left(y^{\prime}\right)\right]$. Then we apply Lemma 6.4 to transform $x^{\prime \prime}$ to $y^{\prime}$.

See Figure 1 for the deformation of a square to a parallelogramm via two bendings.

Remark 6.6. The rotation angle $\gamma$ depends continuously on the parameter $r$. The angle $\gamma$ can be arbitrary close to zero as $r$ approaches the walls $\Sigma$ or the boundary of the polyhedron $\mathcal{D}_{4}$. This corresponds to a degeneration of the elliptic curve. In the limit the birational transformation $\theta$ will have isolated fixed points: singular points of the curve $E$.

\subsection{Deformations of $n$-gons}

Lemma 6.7. Suppose that $Q \in M_{r}$ is a nondegenerate n-gon. Then there exists a diagonal $d$ of $Q$ such that the bending in $d$ changes the distance between at least two vertices $A, B$.

Proof. Suppose that $Q \in M_{r}$ is a $n$-gon and $M_{r}$ is a nonsingular moduli space. Our problem is to deform $Q$ via bending so that the distance between two distinct vertices $A=v_{1}, B=v_{s}$ of the polygon $Q$ is changing (assuming that $|s-1| \neq 0,1$ ). If the distance $|A B|$ does not change under bending in a diagonal $\left[v_{k}, v_{s+i}\right]$ then either $A$ or $B$ belong to the line $\left(v_{k}, v_{s+i}\right)$.

Suppose that the distance $|A B|$ does not change for any choice of 


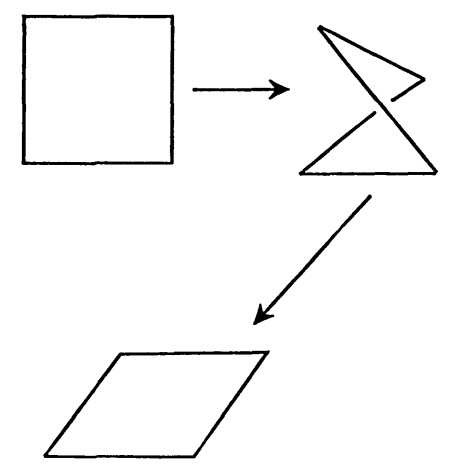

FiguRE 1

$s, k$ above. Then either $A$ or $B$ belong to the line $\left(v_{k}, v_{s+i}\right)$ for all $1<k<s, 0<i \leq n-s$. This means that either all the vertices of $Q$ except $A$ belong to a single line $\left(v_{2}, B\right)$ through $B$ or all the vertices of $Q$ except of $B$ belong to a single line $\left(v_{2}, A\right)$. (The polygon $Q$ must be a pseudo-triangle.) Suppose that the former case takes place. Then instead of vertices $A, B$ we choose say $v_{2}, v_{n}$ and applying bending along the diagonal $[A B]$ we can change the distance $\left|v_{2} v_{n}\right|$.

Suppose that $f: S^{1} \rightarrow \mathbb{R}$ is a continuous function. Then we define

$$
\operatorname{var}_{t \in S^{1}}(f)=\left|\max _{t}(f)-\min _{t}(f)\right|
$$

to be the variation of the function $f$ on the circle $S^{1}$.

We recall that $\Psi_{d}^{t}$ denotes the normalized bending in the diagonal $d$ of a polygon $P \in M_{r} ;|d|$ denotes the length of this diagonal.

Definition 6.8. Suppose that $P \in \mathcal{P}_{n}$ and $\pi(P) \notin \Sigma$. We define the following function

$$
\begin{gathered}
\delta(P)=\max \left\{|d| \operatorname{var}_{t \in S^{1}}\left(\left|\Psi_{d}^{t}(A), \Psi_{d}^{t}(B)\right|\right): A, B \text { are vertices of } P,\right. \\
d \text { is a diagonal of } \mathrm{P}\}
\end{gathered}
$$

A pair of diagonals $([A, B], d)$ providing this maximum will be called a maximal pair.

It is clear that the function $\delta$ is continuous. Thus by Lemma 6.7 for any compact $K \subset D_{n}-\Sigma$ there is a number $\epsilon_{K}>0$ such that $\delta(P) \geq \epsilon_{K}$ for each $P \in \pi^{-1}(K)$. 


\section{Proof of Theorem 6.2}

We have already proved Theorem for quadrilaterals, thus we can assume that the number $n$ of vertices is at least 5 . Arguing by induction we can assume that Theorem is valid for all spaces of $k$-gons, where $4 \leq k<n$.

Step 1. Take a n-gon $P \in M_{r}$. The space $M_{r}$ is nonsingular, thus $\delta(P) \geq \epsilon>0$ where $\epsilon$ depends only on $r$. Choose a pair of vertices $A, B$ and diagonal $d$ of $P$ which maximize the function $\delta(P)$. The maximal variation $\operatorname{var}_{t \in S^{1}}\left(\left|\Psi_{d}^{t}(A), \Psi_{d}^{t}(B)\right|\right)$ is at least $\epsilon$ since the length of the diagonal $d$ is at most 1 . Split $P$ along the diagonal $[A, B]$ in two polygons $P^{\prime}, P^{\prime \prime}$ treating $[A, B]$ as a new side with fixed length. There is at most $n ! / 2$ of "bad" values of $|A B|$ such that the moduli spaces $M_{r^{\prime}}, M_{r^{\prime \prime}}$ of $P^{\prime}, P^{\prime \prime}$ are singular. (We also include zero in the list of "bad" values.) Denote the number of vertices of $P^{\prime}$ by $n^{\prime}$ and the number of vertices of $P^{\prime \prime}$ be $n^{\prime \prime}$.

Then we use a bending in the diagonal $d$ to deform $P$ to a polygon $P_{*}$ so that the distance from $\left|\Psi_{d}^{t}(A), \Psi_{d}^{t}(B)\right|$ to each of these "bad" values is maximal (which is at least $\epsilon / n !$ ).

The proof of the following proposition is obvious and is left to the reader

Proposition 6.9. Let $C \subset \mathcal{D}_{n}-\Sigma$ be a compact. Then there are two compacts $C^{\prime} \subset \mathcal{D}_{n^{\prime}}-\Sigma, C^{\prime \prime} \subset \mathcal{D}_{n^{\prime \prime}}-\Sigma$ such that for any $P \in \pi^{-1}(C)$ we have

$$
P_{*}^{\prime} \in C^{\prime}, P_{*}^{\prime \prime} \in C^{\prime \prime}
$$

Thus the polygons $P_{*}^{\prime}, P_{*}^{\prime \prime}$ satisfy the property that the function $\delta$ on their moduli spaces $M_{r^{\prime}}=\pi^{-1}\left(\pi\left(P^{\prime}\right)\right), M_{r^{\prime \prime}}=\pi^{-1}\left(\pi\left(P^{\prime}\right)\right)$ is bounded from below by some positive number $\epsilon_{1}$.

Define a relation $R$ in $\left[\mathcal{P}_{n}-\pi^{-1}(\Sigma)\right] \times\left(\{1, \ldots, n\}^{2}\right)^{2} \times\left[\mathcal{P}_{n}-\pi^{-1}(\Sigma)\right]$ as the set of tuples $\left(P,(i, j),(k, s), P^{*}\right)$ where the diagonals $([A, B]=$ $\left.\left[v_{i}, v_{j}\right], d=\left[v_{k}, v_{s}\right]\right)$ form a maximal pair and the polygon $P_{*}$ is obtained from $P$ via bending in the diagonal $d$ as above.

Proposition 6.10. The relation $R$ is closed.

Proof. It is enough to prove that if $\lim _{s \rightarrow \infty} P_{s}=P$ in the space $\mathcal{P}_{n}-\pi^{-1}(\Sigma)$ then for any $\left(P_{s},\left[A_{s}, B_{s}\right], d_{s}, P_{s}^{*}\right) \in R$ and sufficiently large $s$, the pairs $\left(\left[A_{s}, B_{s}\right], d_{s}\right)$ are maximal for the limiting polygon $P$ as well.

Pick a subsequence with constant $\left(A_{s_{k}}, B_{s_{k}}, d_{s_{k}}\right)$. By continuity of the function $\delta$ it is enough to check that the $\left|d_{s_{k}}(P)\right| \neq 0$ for the limiting polygon $P$. The nonvanishing of $\left|d_{s_{k}}(P)\right|$ follows from the inequalities $0 \leq \epsilon \leq \delta\left(P_{s_{k}}\right) \leq\left|d_{s_{k}}\left(P_{s_{k}}\right)\right|$. 


\section{Step 2.}

Lemma 6.11. Let $r \in \mathcal{D}_{k}-\Sigma$. Then for each $i$ the moduli space $M_{r}$ contains a pseudo-triangle $T_{i}=\left(v_{1}, \ldots, v_{k}\right)$ such that $v_{i}$ is a pseudovertex of $T_{i}$. Proof. Assume that the assertion is valid for all $3 \leq k^{\prime}<$ $k$. By renumeration of vertices it is enough to construct the pseudotriangle $Q_{1}$. Since the perimeter of the polygon is normalized to be equal to 2 , there is a pair $\left(r_{j}, r_{j+1}\right)\left(j \in \mathbb{Z}_{k}\right)$ different from $\left(r_{k}, r_{1}\right)$ such that $r_{j}+r_{j+1}<1$. Hence we can apply the same induction argument as Lemma 1 in [10] to find a polygon in $M_{r}$ where $e_{j} \cup e_{j+1}$ forms an edge. As the result of this procedure we construct the required pseudotriangle.

Remark 6.12. The case when $T_{1}=T_{2}$ happens exactly when there is a number $2<s<k$ such that $r_{1}+\ldots+r_{s} \geq 1$ and $r_{s+1}+\ldots+r_{k}+r_{1}>1$.

Recall that $k<n$ and by the induction hypothesis we have a function $\ell=\ell(r)$.

Define the relation $\Theta_{\Delta}$ on $\left(\mathcal{P}_{k}-\Sigma\right)^{2}$ to be the set of pairs $(P, T)$ where: (i) the pseudo-triangle $T$ belongs to the moduli space of $P$ and (ii) $P$ can be deformed to $T$ via at most $\ell(r)$ bendings.

Proposition 6.13. The relation $\Theta_{T}$ is closed in $\left(\mathcal{P}_{k}-\Sigma\right) \times \mathcal{P}_{k}$ and its projection to the first factor is onto.

Proof. Since we consider only parameters $r \notin \Sigma$ the equality $r_{j}+$ $r_{j+1}=1$ (in the proof of Lemma 6.11) is impossible. Thus the relation on $\left(\mathcal{P}_{k}-\Sigma\right) \times \mathcal{P}_{k}$ given by the condition (i) is closed. The statement of the Proposition follows from the induction hypothesis in Theorem 6.2.

This lemma together with Lemma 6.11 implies that arguing by induction we can deform via bending each of polygons $P_{*}^{\prime}, P_{*}^{\prime \prime}$ to pseudotriangles $T^{\prime}, T^{\prime \prime}$ keeping the length $|A B|$ fixed so that $A$ is a pseudovertex of $T^{\prime \prime}$, and $B$ is a pseudo-vertex of $T^{\prime}$. The number of bendings which we have to use here is bounded from above by a function which depends on $r^{\prime}, r^{\prime \prime}$ only. In the case when both $A, B$ are pseudo-vertices of $T^{\prime}$ and $T^{\prime \prime}$ these pseudo-triangles form a quadrilateral and we can go directly to the Step 3 (see Figure 2). Assume that $B$ is not a pseudovertex of $T^{\prime \prime}$ and $A$ is a not a pseudo- vertex of $T^{\prime}$ (see Figure 2). The triangles $T^{\prime}, T^{\prime \prime}$ form a hexagon, split it along the diagonal $d^{\prime}=\left[X, Y^{*}\right]$ into two quadrilaterals $S^{\prime}, S^{\prime \prime}$, where $d^{\prime}$ is a side of fixed length. Then the triangle inequalities imply that both $S^{\prime}, S^{\prime \prime}$ can be deformed to pseudotriangles $L^{\prime}, L^{\prime \prime}$ where $X, Y^{*}$ are pseudo-vertices. This again gives us a quadrilateral. Thus we can go to the Step 3.

In the remaining case when $A$ is not a pseudo-vertex of $T^{\prime}$ and $B$ is a pseudo-vertex in both $T^{\prime}, T^{\prime \prime}$ we split the polygon formed by $T^{\prime}, T^{\prime \prime}$ along the diagonal $[X, B]$ (see Figure 2) and deform the quadrilateral 


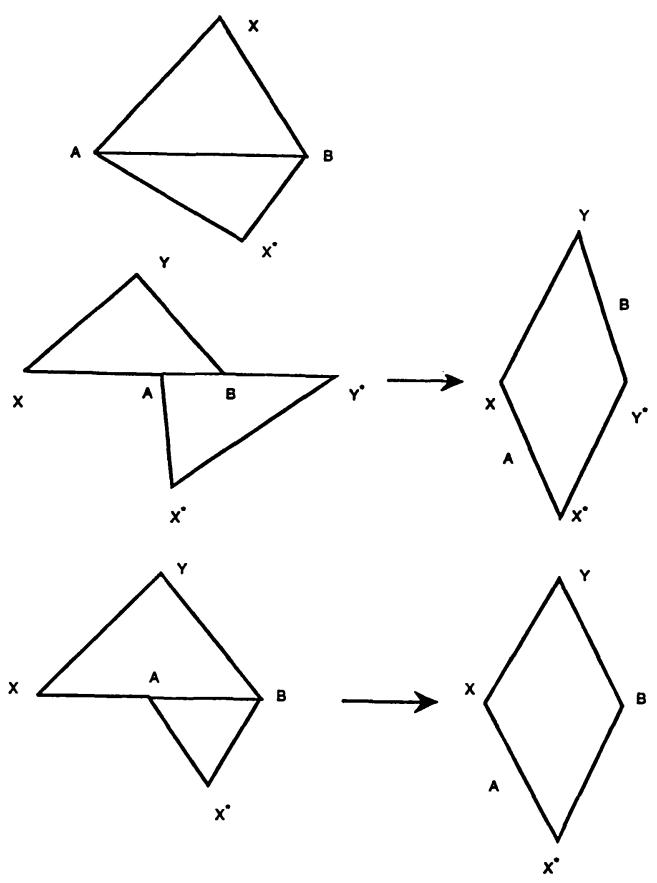

FiguRE 2

$\left[X, B, X^{*}, A\right]$ to a pseudo-triangle with the vertices $X, B, X^{*}$ keeping the triangle $[X, Y, B]$ fixed. Then we again can go to the Step 3 .

Step 3. As the result of Step 2 we deform the polygon $P$ to a polygon $Q$ which is the union of two pseudo-triangles $\Delta^{\prime}, \Delta^{\prime \prime}$ minus the diagonal $[A, B]$.

Remark 6.14. As before the relation $\Theta$, which consists of the pairs $(P, Q)$ above, is closed in $\left(\mathcal{P}_{n}-\Sigma\right) \times \mathcal{P}_{n}$.

The moduli space of the quadrilateral $Q$ is nonsingular since $r \notin \Sigma$. We again apply the induction to $\operatorname{deform} Q$ so that it becomes a pseudotriangle $T$. It follows from the induction hypothesis that the relation $\Xi$, which consists of pairs $(Q, T)$ as above, is closed in $\left(\mathcal{P}_{4}-\Sigma\right) \times \mathcal{P}_{4}$.

Thus we have proved the assertion (a) of Theorem 6.2 for $n$-gons. Namely, for each polygon $P$ we have constructed a piecewise-smooth bending curve $\gamma(P) \subset M_{r}$ which connects the polygon $P$ with a pseudotriangle $T$. Each smooth arc of this curve is given by bending in one of diagonals. (However the curve $\gamma$ is not necessarily unique.) The fact that the function $\ell(r)$ (the number of bendings) is bounded on compacts 
follows from the induction hypothesis via Proposition 6.9. This implies the assertion (b) of Theorem 6.2.

For a pseudo-triangle $T \in M_{r}$ denote by $Y(T)$ the subset in $M_{r}$ consisting of those polygons $P$ such that at least one of the bending curves $\gamma(P)$ terminates at $T$. Thus the relation $\{(P, T): P \in Y(T)\}$ is the composition of closed relations $R, \Theta, \Xi$. This implies that each $Y(T)$ is closed and the assertion (c) follows.

It remains to prove the assertion (d). The closed subsets $Y(T)$ can intersect. We say that two pseudo- triangles $T_{1}, T_{2}$ are equivalent if $Y\left(T_{1}\right) \cap Y\left(T_{2}\right) \neq \emptyset$. This generates an equivalence relation on the finite set of pseudo-triangles in $M_{r}$. The space $M_{r}$ is connected and all $Y(T)$ are closed sets; thus all pseudo-triangles are mutually equivalent. Hence any polygon $P$ can be deformed to a pseudo-triangle via at most $\ell(r)$ bendings (by the assertion (a)) and any two pseudo-triangles can be deformed to each other via at most $n^{3} \ell(r)$ bendings. This finishes the proof.

\section{Acknowledgements}

We would like to thank Mark Green for showing us how to prove Lemma 6.3 before [6] came to our attention. We would also like to thank Janos Kollar, Yi Hu, Valentino Zocca and David Rohrlich for helpful conversations.

\section{References}

[1] J. Arms, M. Gotay \& G. Jennings, Geometric and algebraic reduction for singular momentum maps, Advances in Math. 79 (1990) 43-103.

[2] T. Delzant, Hamiltoniens periodiques et images convexes de l'application moment, Bull. Soc. Math. France 116 (1988) N 3, 315-339.

[3] A. Douady, C. Earle, Conformally natural extension of homeomorphisms of the circle, Acta Math. 157 (1986) 23-48.

[4] P. Deligne, G. Mostow, Monodromy of hypergeometric functions and nonlattice integral monodromy, Publications of IHES, 63 (1986) 5-90.

[5] M.L. Green, private communication.

[6] C.G. Gibson, P.E. Newstead, On the geometry of the planar 4-bar mechanism, Acta Applicandae Math. 7 (1986) 113-135.

[7] W.M. Goldman, Invariant functions on Lie groups and Hamiltonian flows of surface group representations, Invent. Math. 85 (1986) 263-302.

[8] W. Goldman, J.J. Millson, The deformation theory of representations of fundamental groups of compact Kähler manifolds, Publ. Math. of IHES, 67 (1988) 43-96. 
[9] L. Jeffrey, J. Weitsman, Torus actions, moment maps and the moduli space of flat connections on a two-manifold, Preprint.

[10] M. Kapovich, J.J. Millson, On the moduli space of polygons in the Euclidean plane, J. Differential Geom. 42 (1995) 133-164.

[11] _ The relative deformation theory of representations of flat connections and deformations of linkages in constant curvature spaces, Compositio Math. (to appear).

[12] G. Kempf, L. Ness, The length of vectors in representation spaces, In: Algebraic Geometry, Proceedings, Copenhagen 1978, Springer Lecture Notes in Math. 732 233-244.

[13] F. Kirwan, Cohomology of Quotients in Symplectic and Algebraic Geometry, Mathematical Notes, Princeton University Press, 1984.

[14] A. Klyachko, Spatial polygons and stable configurations of points on the projective line, Algebraic geometry and its applications (Yaroslavl, 1992), p. 67-84.

[15] S. MacLane, Homology, Die Grundelheren der Mathematischen Wissenschaften, 114 (1967), Springer-Verlag.

[16] J.J. Millson, B. Zombro, A Kähler structure on the moduli space of isometric maps from the circle into Euclidean space, Inventiones Math., to appear.

[17] J.J. Millson, B. Zombro, in preparation.

[18] D. Mumford, J. Fogarty, F. Kirwan, Geometric Invariant Theory, Third Enlarged Edition, Ergebnisse der Mathematik und Ihrer Grenzgebiete 34, Springer-Verlag, 1994

[19] L. Ness, A stratification of the null cone via the moment map, Amer. Journal of Math. 106 (1984) 1281-1329.

[20] W. Thurston, Shapes of Polyhedra, Preprint, 1987.

[21] K. Walker, Configuration spaces of linkages, Undergraduate thesis, Princeton Univ., 1985.

[22] J. Weitsman, Real polarization of the moduli space of flat connections on a Riemann surface, Comm. Math. Phys. 145 (1992) p. 425-433.

\author{
UNIVERSITY OF UTAH \\ UNIVERSITY OF MARYLAND
}

